\title{
GOTEN UND INGVAEONEN.
}

$N_{\text {ach der stellung, die Tacitus sowohl als ibre eigene }}$ stammsage den Goten einräumt, waren diese ursprünglich die nächsten nachbarn der Rugen und der lugischen stämme, standen aber auch von den nördlicheren Bastarnen nicbt allzu ferne. Die bedingungen gleichartiger sprachentwicklung waren also hier gegeben, umsomehr als von der grenze Mährens her durch lugisches gebiet hindurch zu den Goten und der bernsteinküste ein lebhafter handelsverkehr bestand. Auch nach ihrem aufbruch aus den alten stammsitzen waren, von den westwärts abziehenden lugischen Burgunden abgesehen, die Lugier, Goten, Rugen und Skiren in engerer verbindung, zudem galt bei ihnen allen das gotische des Wulfila als kirchensprache und ihr christentum war arianisch. Man sieht, wieso Procopius dazu kam, sowohl Vandalen (De b. Vand. 1, 2) als Skiren (De b.

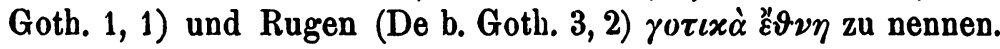
Grösseres gewicht ist aber auf diesen ausdruck schon deshalb nicht zu legen, weil derselbe gewährsmann, De b. Goth. 1, 1 und De b. Vand. 1, 3 sogar die iranischen Alanen - doch böchstens wegen ibres gotischen christentums und ibrer engeu politischen verbindung mit den Vandalen - mit unter die gotischen stämme rechnet. Dass in wahrheit weder die Vandalen, noch die Skiren, noch die Rugen unter den begriff Goten fallen, liegt so klar zu tage, dass es gar nicht erst nötig ist, die gründe dafur besonders aufzuzählen.

Es ist sogar fraglich, ob wir berechtigt sind, von einer vandilischen völkergruppe zu sprechen und dabei Rugen und Goten mit einzubegreifen. Die eigene sagentuberlieferung der letzteren stellt sie schon in ihrer urzeit und in ihrer alten 
heimat an der unteren Weichsel zu den Ulmerugen und den Vandalen in gegensatz. Nachdem eben von ihrer einwanderung aus Scandza die rede war, heisst es von ihnen bei Jordanes c. 4 weiter: unde mox promoventes ad sedes Ulmerugorum, qui tunc Oceani ripas insidebant, castra metati sunt eosque commisso proelio propriis sedibus expulerunt eorumque vicinos Vandalos iam tunc subiugantes suis applicavere victoriis. Nachbarn der Rugen waren die Vandalen in dem späteren eingeschränkten sinne dieses namens gar nicht, und umsoweniger empfiehlt es sich, hier schon diesen vorauszusetzen. Auf der anderen seite steht freilich das zeugnis des Plinius, der HN. 4 §99 die Gutones als einen teil der Vandili aufführt. Was dagegen Rugen und Lemovier betrifft, wird sich gar nichts beibringen lassen, was für ihre zugehör zu den Vandalen sprechen könnte. Auch die Goten bilden mindestens unter diesen eine besondere gruppe; denn $z \mathfrak{u}$ den Lugiern gehören sie auf keinen fall: vgl. Tacitus, Germ. 43: ultra Lygios Gotones regnantur. Und wenn ibre stammsage Scandza als ihre älteste heimat angibt, aus der sie $\mathrm{zu}$ schiff uber das meer gekommen seien, so verdient sie umsomehr glauben, als in Skadinavien selbst - gegenuber der Weichselmundung - ein stamm namens Goten, altn. Gotar, fortbesteht, derjenige, von dem die insel Gotland, altn. Gotland, Gotaveldi, den namen hat.

Ueber die bedeutung des Gotennamens weiss Wrede, Spr. d. Ostg. 44 nichts besseres zu sagen, als dass, wer die ZeussGrimmsche deutung der 'Wandalen' als der 'umherziehenden' annehme, auch gegen die 'Goten' als die 'ausgebreiteten' kein bedenken haben werde. Wir haben indes jenen 'umherziehenden Vandalen' schon ein für alle mal den laufpass gegeben und können uns daher auch mit den 'ausgebreiteten Goten' nicht weiter befassen. Aber schon Lottner KZ. 5, 154 war auf dem richtigen wege und irrt nur, wenn er durch aisl. goti 'hengst' verleitet für den namen Goten sowohl als auch für das aisl. appellativ golnar, das in poetischer sprache 'männer, helden' bedeutet, eine ältere bedeutung 'hengste' voraussetzt und furr das gleichnis an aisl. jofurr 'fürst' erinnert. Der hengst ist möglicherweise als der 'besprenger' goti genannt: vgl. aisl. gota in dem sinne 'to spawn', got n. 'spawning', gota, u f. 'spawn', gotrauf f. 'the spawn hole in female codfish or salmon', gotungr 
m. 'young fish, fry' (Cleasby-Vigfússon 202. 209); wahrscheinlicher aber ist das wort, das nur bei skalden belegt ist, wie Bugge, Tolkning af runeskriften på Rökstenen $136 \mathrm{f}$. annimmt, von haus aus der eigenname von Gunnars ross und als solcher vom volksnamen ausgegangen. Aber auch wenn die andere erklärung die richtige sein sollte, wird dasselbe wort unmittelb ar zur bedeutung 'zeugungskräftiger mann' gelangt sein, wie denn auch norweg. gūt 'junger mann, bursch' und aisl. gautar unter anderem 'viri' (Egilsson 226) bedeutet; und wenn Oðinn nicht nur Gautr Gauti allein, sondern so wie aldafaðir so auch aldagautr heisst, worin der erste teil der gen. plur. von old ist, bezeichnet ihn dieser name wohl als den 'schöpfer', den 'erzeuger der menschen'. Somit sind sowohl Goten als Gauten durch ihren namen als die 'zeugungskräftigen' gekennzeichnet, sofern ihnen derselbe nicht etwa erst in dem sinne 'männer, helden' beigelegt wurde. Eine namensform got. Gudans oder Gupans hat es nie gegeben; aisl. Godpjód ist, wie Heinzel Hervararsaga 76 (WSB. 1887, 490) gezeigt hat, in folge von tonlosigkeit der ersten silbe aus Gotpjód entstanden.

Wenn uns Ptolemaeus 2, 11,16 als die beiden südlichsten stämme der grossen $\Sigma x \alpha v \delta i ́ \alpha$ die $\Gamma$ oṽ $\tau \iota$ und die $\Delta \alpha v x i o v \varepsilon \varsigma$ namhaft macht, so wird man dabei an die Gauten und Dänen zu denken haben. Zeuss s. 158 hat ktihn genug Aavxíoves in $\Sigma x \alpha \nu \delta i o v \varepsilon s$ ändern wollen; allein dieser name, dem got. gadauka 'hausgenoss' zur seite steht, ist zweifellos sehr gut überliefert. Umsomehr kommt es in frage, ob man Гoṽ $\alpha \iota$ unter voraussetzung eines nicht recht begreiflichen feblers in $\Gamma \alpha \tilde{v} \tau \alpha \iota$ ändern darf. Wenn es neben dem appellativ *gautaz (= aisl. gautr, ahd. -gôz und ags. -zéat in namen) ein gleichbedeutendes

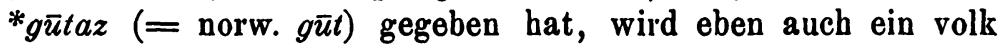
* Gautōz und * Gūtōz geheissen haben können; ja selbst die möglichkeit eines parallelnamens *Gutōz neben *Gautōz kommt in betracht. Für die festländischen Goten ist es mindestens wahrscheinlich, dass sie * Gutans und * Gutôs geheissen haben; aber freilich lässt sich die letztere namensform nur mit Sievers in Pauls Grundr. 1, 407 aus den in späteren griech. und lat.

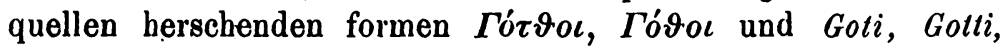
Golhi, keineswegs aber mit Wrede, Spr.d. Ostg. 46 auch aus got. Gutpiuda und aus dem neben Gotna vorkommenden genitiv 
Gota von aisl. Gotar erschliessen. Wenn wir aber auch für jene $\Gamma o \tilde{\tau} \alpha \iota$ bei ${ }^{*} G \bar{u} \iota \bar{o} z$ stehen bleiben, so zeigt sich doch damit schon, dass es hier so wenig wie bei den $* p_{\text {uronez, die }}$ auch * Ermundurōz und * Puringōz hiessen, oder bei der gotischen unterabteilung der * Greutungôs, * Grautungôs oder *Grutungôs (s. Wrede, Spr. d. Ostg. 49) auf eine feste form des namens, sondern dass es vielmehr auf den begriff desselben ankam. Und sollten nicht sowohl *Gautōz und *Gūtōz als auch *Gutonez und ${ }^{*} G u t \bar{z} z$ urspringlich alles namen ein und desselben stammes gewesen sein? Als sich teile desselben, von den übrigen durch das meer geschieden, selbständig entwickelten, konnte die mehrheit der vorhandenen namen leicht verwendet werden, um unterscheidende bezeichnungen zu schaffen. Diese erwägungen führen darauf, dass es nicht notwendig die Goten auf Gotland, sondern dass es möglicherweise die Gauten waren, von denen die Weichselgoten ausgiengen. Und vielleicht stehen gar die gotischen Greutungi mit den Evagreotingi des Jordanes in zusammenhang, die Müllenhoff, der (s. $63 \mathrm{f}$.) ihren namen aus dem verderbten Evagre Otingis der hss. hergestellt hat, im siiden des Wettersees ansetzt.

Dies hätte allerdings zur voraussetzung, dass das gotische gesammtvolk schon in seiner skadinavischen urheimat in mehrere stämme zerfiel und zwar ganz oder teilweise dieselben, die uns nachmals an der Donau und am Pontus entgegentreten. Plinius, Tacitus und Ptolemaeus sprechen allerdings nur von Goten schlechtweg; aber wer wollte daraus schon folgern, dass die stammeseinteilung dieses volkes zu ihrer zeit noch nicht bestand?

Wie viele Gotenstämme wir zu unterscheiden haben, lehrt die sage, wenn sie von drei fahrzeugen erzählt, auf denen die überfahrt aus Scandza erfolgt sei und aus deren einem, das zuletzt das ziel erreichte, die trägen Gepiden hervorgegangen seien. Aus den beiden anderen schiffen hat man offenbar die Tervingen (Visegoten) und Greutungen (Ostrogoten) hergeleitet. Der bestand eines vierten Gotenstammes ist aber dadurch ausgeschlossen. Keinesfalls wird man also die Taifalen (oder Thaifalen?) mit Müllenhoff s. 91 als solchen zu betrachten haben, vielmehr als eine lugische oder bastarnische abteilung. Auch die a.a. o. vorgetragene erklärung ihres namens, den sie 
ohne zweifel nach ihrer niederlassung in der 'dakischen ebene' erhalten haben sollen, ist in hohem grade bedenklich.

Wenn uns Jordanes c. 17 den namen Gepidoios d.i. -aujôs (Müllenhoff s. 365) uberliefert, so sind wir dadurch über die sitze der Gepiden wenigstens einigermassen unterrichtet. Ueber die stellung der Ostrogoten und Visegoten wlirden uns ihre namen aufklären, wenn es sich nur erweisen liesse, dass sie schon an der unteren Weichsel aufgekommen sind. Auch die frage, wie weit sich dort der germanische bereich nach osten hin erstreckte, bleibt vorläufig unerledigt, bis uns etwa funde eine aufklärung bringen. Von den flüssen im osten der Weichsel ist Guthalus bei Plinius $4 \S 100$ entschieden von den Goten benannt, 'wenn auch nicht gerade nach ihrem volksnamen', wie Müllenhoff s. 209 mit recht betont. Der name setzt ein zu giutan gebildetes adjectiv got. *gutals, germ. *gutalaz voraus, bis auf den unwesentlichen suffixablaut dasselbe, das dem isl. gutla 'to gurgle, used of the noise made by a liquid when shaken in a bottle' (Cleasby-Vigfússon 221) zu grunde liegt. Den namen Xóvos bei Ptolemaeus 3, 5, 1 hat Múllenhoff s. 351 durch hinweis auf aisl. hrynja 'herunterfallen' von gewässern 'herabsttlrzen, strömen', ahd. runen, mhd. rünen befriedigend erklärt. Er ist entweder ebenso wie Guthalus ein deutscher name für den Pregel oder Guthalus und Xóvos verteilen sich auf Pregel und Alle. 'Pov́doov, bei Ptolemaeus der name des nächst östlichen flusses nach dem Xoóvos, also ohne zweifel der Memel, bedeutet so viel als 'der rote'; doch ist es nicht entscheidbar, ob das wort gotisch oder aistisch ist; vgl. lit. rùdas 'rotbraun' und das seiner gestalt und seinem ursprunge nach mit 'Pov́doov völlig zusammenfallende lit. rudi 'herbst', anderseits aber mhd. rŏt 'rot', roten 'rot werden' (neben rôt und rôten), ahd. rutichôn 'rötlich sein', ags. rudu 'röte' rudiz 'rot'. Wenn der rechte und bedeutendste mundungsarm der Memel lit. Rusne heisst, darf man deshalb 'Pov́doov nicht in 'Pov́oøv ändern wollen. Gleichwohl wird zwischen beiden namen ein zusammenhang bestehen, denn auch Rusne ist kaum etwas anderes als 'der rote' oder 'der rostfarbene'; vgl. lett. rusa 'rost', ahd. rosamo 'rubor', aerugo, lentigo' und got. *birusnjan 'verehren' (Kluge EW.4 284), das ist wohl 'rot werden vor jemandem'. Wenn aber auch von den Goten vielleicht 
aus ihrer eigenen sprache verstanden, sind Rusne und 'Pov́dwv von haus aus wohl eher aistisch; befand sich doch die bernsteinkliste nach Tacitus, Germ. 45 in den händen der Aisten, nicht in denen der Goten; vgl. Müllenhoff s. 19.

Grenze der Goten gegen westen ist bei Ptolemaeus die Weichsel. Ueberschreiten wir diese in der angegebenen richtung, so stossen wir bei ibm an der meeresk tiste zunächst auf das

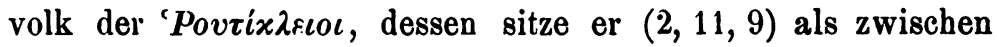

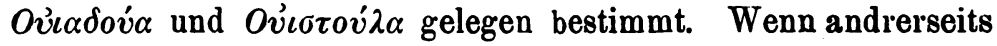
die gotische wandersage bei Jordanes c. 4 von kämpfen der Goten mit den Ulmerugi, d. i. Inselrugen auf den Weichselholmen (Müllenhoff s. 5), berichtet, und Tacitus, Germ. 43 nach den Goten längs des Oceans Rugii et Lemovii aufzählt, so leuchtet es ein, dass unter jenen 'Povtix stehen sind. Dazu kommt, dass Ptolemaeus in ibrem gebiet, wenn auch hart an dessen westgrenze, einen ort 'Pov́rıov ansetzt. Wenn dagegen Zeuss s. 154 die Rugen in den $\Sigma \varepsilon \iota \delta \iota v o i$ ( $\Sigma \iota \delta \iota v o i ́)$ des Ptolemaeus widererkennen möchte, die doch an die Goten gar nicht hinanreichen, hat dies lediglich darin seinen

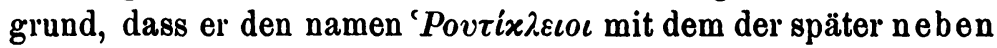
den Rugen auftretenden Turkilingen verbinden zu durfen glaubt. Aber die dabei vorausgesetzte zweimalige buchstabenumstellung,

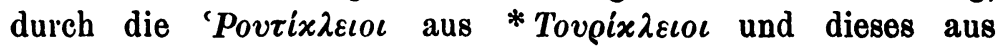
* Tovoxi $2 \varepsilon \iota 0$ entstanden sein soll, ist wenig wahrseheinlich; und wie wäre zudem der auslaut in * Tovoxideloc gegenuber Turcilingi zu beurteilen? POYTIKAEIOI ist doch sicher aus POYГIKAEIOI verderbt und zeigt uns den anderen gebräuchlicheren namen des volkes mittels eines deminutivsuffixes weitergebildet: zu germ. ${ }^{*}$ Rugīz verbält sich $*$ Rugiklīo gerade wie alts. nessikin 'würmchen' zu nesso 'wurm' oder ahd. gensi(n)$k \hat{\imath}(n)$ 'gänschen' zu gans; luber die ableitung s. Kluge, Nom. stammbild. $\S 63$. Dass die antike umschrift dem namen nach dem vorbilde anderer volksnamen masculine endung gab, ist nicht auffällig. Durch das verständnis seines suffixes ist uns auch seine deutung insoferne erleichtert, als er, wenn er der verkleinerung fähig ist, nur substantivischer, nicht adjectivischer herkunft sein kann. Ags. Holmry(б)um Widsið 21 (dem freilich 
Ruzum 69 gegentabersteht), altn. Rygir, langob. Rugiland scheinen auf einen $i$-stamm * Rugi- hinzuweisen. Der volksname in seiner einfacheren gestalt wüde sich dann völlig decken mit ags. ryze, engl. rye, aisl. rugr 'roggen', aber auch allein mit diesem worte. Dadurch wird er freilich noch nicht verständlich, allein das verwante lit. rugýs bedeutet 'roggenkorn' und erst im plural (rugei) 'roggen', so dass auf germ. und slav. seite - vgl. slav. rǔž 'roggen' - eine bedeutungsentwickelung ähnlich wie bei unserem korn vorliegen durfte. Ein volksname des sinnes 'die körner' und 'die körnlein' ist allerdings noch immer sonderbar; aber vielleicht hat eine geschichte dazu gehört, die ihn uns verständlich machen würde. Je seltsamer er tibrigens ist, desto unwahrscheinlicher ist es, dass er in Deutschland und in Norwegen selbständig aufgekommen ist; vielmehr werden die Rugii an der Weichsel und die Rygir am Bukkefjord nur durch wanderung getrennte teile eines und desselben volkes sein, uber dessen ältere sitze wir freilich ganz im unklaren sind.

An die 'Pov $(\gamma) i x \lambda \varepsilon \iota 0 \iota$ schliessen sich auf der ptolemaeischen karte in der richtung gegen westen die $\Sigma \iota \delta \iota v o i$ und weiterbin die $\Phi \alpha \rho \alpha \delta \varepsilon \iota \nu \circ i$ oder $\Phi \alpha \rho o \delta \varepsilon \iota v o l$ an. Beide stämme sind unter sich und von ihren nachbarn durch flüsse geschieden: $\mu \varepsilon \tau \dot{\alpha} \delta \dot{\varepsilon}$

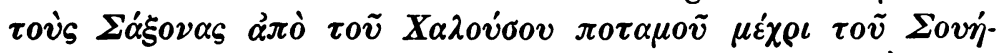

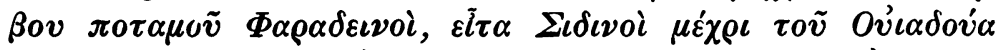

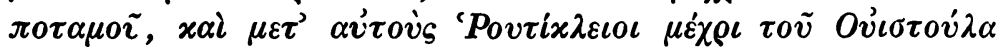
$\pi о \tau \alpha \mu о \tilde{v}$. Sowie der letztgenannte fluss ist auch der $\Sigma o v \tilde{\eta} \beta o \varsigma$ $\pi o \tau \alpha \mu \dot{s}_{s}$ sofort bestimmbar, und zwar als die Oder, da seiner 2, 11, 8 als des grenzflusses zwischen Semnonen und Burgunden gedacht wird, was nicht gut auf einen unbedeutenderen kustenfluss passen wurde. Auch die gradangaben fur seine mundung im verhältnis zu denen für die Weichselmündung und für die einbuchtung an der ostseite der kimbrischen halbinsel sowie

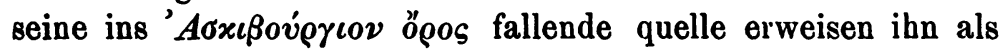
die Oder. Sein name macht jedoch schwierigkeiten. Denn die Oder ist gar nicht ein Svebenfluss $x \alpha \tau^{\prime}{ }^{\prime} \xi \xi \chi \eta^{\prime} \nu$, weit eher noch ein vandalischer, da nur an ihrem mittellaufe, dort wo sie das gebiet der Silingen verlässt, etwa von der mlindung des Bober an, svebische Semnonen ihre anwohner sind, übrigens nur an ihrem linken ufer. Auch dass es $\Sigma o v \tilde{\eta} \beta o s$ und nicht 
$\Sigma o v \eta \beta \iota x o_{s} \pi 0 \tau \alpha \mu o ́ s$ heisst, befremdet auf den ersten blick und bringt uns fast dahin, den namen unmittelbar aus dem verbaladjectiv *swēebiz, also als 'den schläfrigen', zu verstehen, was ja kein unpassender sinn wäre. Allein, wenn uns auf der Tab. Peut. ein fl. Patabus an stelle der Waal begegnet,

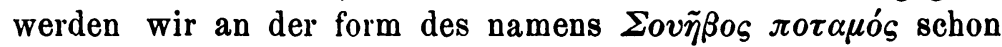
nicht mehr anstoss nehmen, und was die irrtimliche benennung des flusses nach den Sveben betrifft, so ist an das mare Suebicum, d. i. die Ostsee, bei Tacitus, Germ. 45 und an den Oひz$\nu \varepsilon \delta$ xós xó $\lambda \pi \varsigma_{\varsigma}$ bei Ptolemaeus 3, 5, 1 zu erinnern.

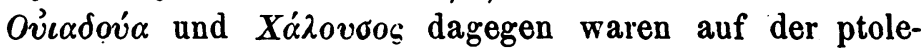
maeischen karte sicher nur als kutstenflusse eingetragen, da sie nirgends bei bestimmung der lage weiter landeinwärts wohnender stämme benutzt sind und auch uber ihre quellen nichts verlautet. Beide namen sind wohl nur, weil sie grenzflussen zukamen, den alten bekannt geworden. Und zwar wird man bei der Ovicadovia, deren mündung nach den gradangaben

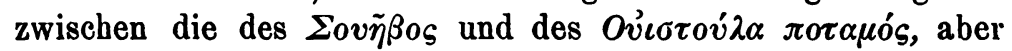
etwas näher bei letzterer angesetzt ist, an Grabow, Wipper oder Persante zu denken haben. Dass sie widerum die Oder selbst, oder gar deren name aus älterem Viadua, ${ }^{*}$ Wiadwō von den Slaven umgeformt sei, wie unbegreiflicher weise Mullenhoff s. 209 und Zeuss s. 16 vermuten, ist bestimmt abzuweisen, weil nicht die geringste spur vorhanden ist, dass die angaben des Ptolemaeus uber die germanische Ostseek üste und ihre völker mehrere ineinander geschobene diathesen darstellen. Der name des $X_{\alpha}^{\prime}$ 'zovøos ist an zwei stellen uberliefert und hat, verglichen mit flussnamen wie Filusa, Suulmusa, ein allzugut germanisches aussehen, als dass man dabei mit Mullenhoff s. 212 an verderbnis aus *Xóßov2os denken möchte; ja vielleicht ist er geradezu eine entsprechung zu dem italischen flussnamen Calore, lat. Calor, d. i. 'der warme fluss'. Seine gleichsetzung mit der Havel würde aber auch allem widersprechen, was wir sonst von ibm wissen. Das gilt tibrigens ebenso von den versuchen, ihn fur die Eider oder die erst nach dem ort Hale benannte Halerau auszugeben: mundet er doch bei Ptolemaeus in die Ostsee. Da sein name eine anknlipfung an einen fortlebenden flussnamen des in betracht kommenden bereiches nicht gestattet, sind wir ausschliesslich auf die gradangaben angewiesen, durch 
die Ptolemaeus seine muindung bestimmt, wobei aber in betracht kommt, dass diese in den verhältnismässig am besten gezeichneten teil seiner karte von Germanien fällt. Nach diesen gradangaben aber, oder vielmehr nach ihrem verhältnis zu denen für die $\Sigma o v \tilde{\eta} \beta o s$, d. i. die Odermündung, und die

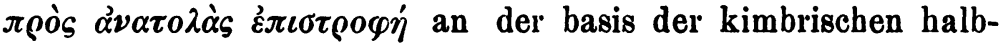

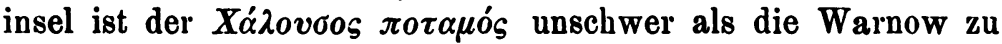
erkennen.

Die wohnsitze der ptolemaeischen $\Sigma \iota \delta \iota v o i$ und $\Phi \alpha \rho \alpha \delta \varepsilon \iota v o i$ sind also recht gut bestimmbar. Es fragt sich nur, wie sich die Lemovii des Tacitus $\mathrm{zu}$ ihnen verhalten. Von vornherein kommen dabei verschiedene möglichkeiten in betracht. Ent-

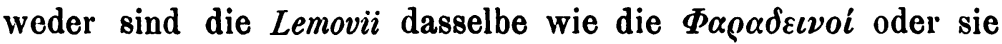
umfassen auch die $\Sigma \iota \delta \iota v o i$ oder gar auch noch die kleineren stämme, die Ptolemaeus im norden der Semnonen namhaft

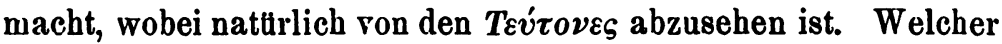
von diesen fällen vorliegt, wäre leicht $\mathrm{zu}$ entscheiden, wenn sich für die $\Phi \alpha \rho \alpha \delta \varepsilon \iota v o i ́$ bei Tacitus anderswo ein unterkommen fände. Von seinen Nerthusvölkern hat freilich keines mit ihnen etwas zu tun, wie eine eingehende untersuchung uber diese noch ergeben wird. Es könnte aber sein, dass sich das machtgebiet der Semnonen zu Tacitus zeit bis an die Ostsee ausgedehnt hatte, und dass er deshalb die völker im norden von ihnen unter ihrem namen mit einbegreift, gerade wie die Parmen, Adraben, Rakaten und Baimen unter dem namen der Markomannen und Quaden. Sehliesslich kann auch ohne solchen grund ein stamn tibergangen sein. Sind doch auch die Tubanten, Chattuarier (Marsen) und Ampsivarier in der Germania des Tacitus nicht erwähnt, von Dänen und Gauten nicht zu sprechen.

Dass die namen $\Phi \alpha \rho \alpha \delta \varepsilon \iota v o i ́$ bei Ptolemaeus und Suardones bei Tacitus und auch die beiden stämme selbst trotz Zeuss s. 154 und J. Grimm GDS. 470 f. nichts mit einander gemein haben, bedarf wohl keiner längeren auseinandersetzung. Nicht viel besser freilich steht es mit Möllers versuch, Ae. volksepos

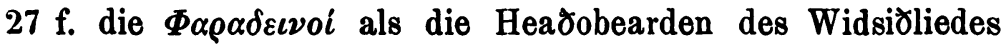
und eine unterabteilung der taciteischen Langobarden $\mathrm{zu}$ erweisen. Der name der Heaðobearden ist nur im gen. plur. als Heaðobeardna (Widsið 49. Beowulf 2032. 2037. 2067) uber- 
liefert, woraus unbedenklich ein nominativ Heaðobeardan zu folgern ist, schon darum, weil *Beardan 'die bärtigen' bedeutet, also einen annehmbaren sinn gibt, während eine bildung Beardnas nicht recht verständlich wäre. Offenbar aber glaubt Möller durch ansetzung des nominativs Heaðobeardnas den

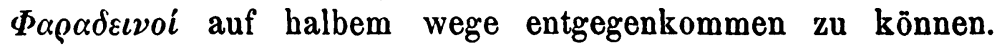

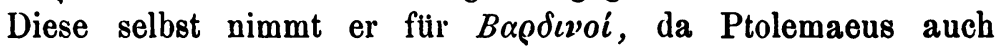

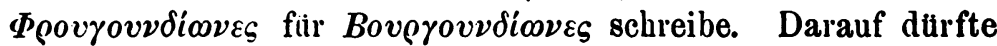
man sich aber nur berufen, wenn gezeigt werden könnte, dass dies wirklich der fall ist. Nach dem was bereits oben s. $40 \mathrm{ff}$. bei besprechung dieser namen gegen die möglichkeit einer umschrift von germ. $b$ durch lat. $f$, griech. $\varphi$ vorgebracht wurde, kann auch $\Phi_{\alpha \varrho \alpha \delta \varepsilon \imath \nu o i}$ nur germ. * Faradīnōz widergeben, es sei denn, dass dabei ein fehler der überlieferung vorliegt. An die möglichkeit eines verderbnisses ist aber höchstens bei dem inlautenden $\Delta$ zu denken, das für $\Lambda$ verschrieben sein könnte. Und neben ahd. wanchalîn, luogalîn, folgalîn, ags. hetlen und zahlreichen anderen ähnlichen bildungen (s. Kluge, Nom. stammbild. $\$ 200$ ), wäre in der tat ein germanisches * faratinaz, oder - da neben $\Phi \alpha \rho \alpha \delta \varepsilon \imath v o i$ eine gleichwertige lesart $\Phi$ $\alpha \rho o \delta \varepsilon \iota v o i$ vorkommt - *farulinaz mit derselben bedeutung wie das cinfachere aisl. forull, d. i. 'rambling, strolling about', sehr wohl möglich. Aber auch cincr bildung * faradinnaz wird man schon wegen got. liuhadeins gut germanisches aussehen nicht abstreiten können.

Die beobachtung, dass namen von nachbarstämmen mittels desselben suffixes gebildet sind, die wir schon bei den Teurisci:

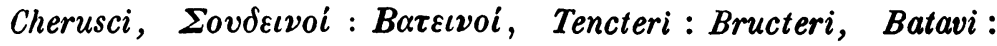

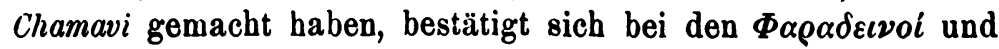

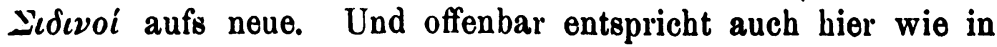
den vorgenannten fällen, soweit wir zu einem verständnis der namen gelangen konnten, der formellen eine bedeutungsbeziehung. Wer darum mit Zeuss s. $154 \Sigma \iota \delta \iota v o i$ von sîda 'seite,

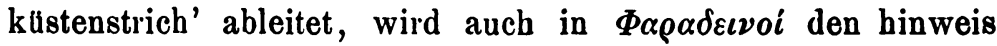
auf einen ortsbegriff suchen mlissen. Dass sich ein solcher in einer ableitung aus der wurzel far leicht entwickeln kann, zeigt unser furt und aisl. fjorðr, ja selbst das holländische vaart, das unter anderem 'kanal, graben, fahrwasser' bedeutet. An fjordähnlichen meeresarmen sassen die $\Phi \alpha \rho \alpha \delta \varepsilon \iota v o i$ zwischen 
Warnow und Oder in der tat, und das könnte uns noch mehr in der vermutung bestärken, dass ihr name wesentlich dasselbc ausdrticke wie der norwegische volksname Firðir. Allein zwei namen, von denen der eine 'klistenanwohner', der andere 'anwohner des fahrwassers' bedeutet, sind schlechte gegenstücke. Zudem fehlt es innerhalb des germanischen nahezu vollständig an anderweitigen belegen für eine verwendung des suffixes -īna-, um örtliche zugehörigkeit auszudrücken, denn von Peucini

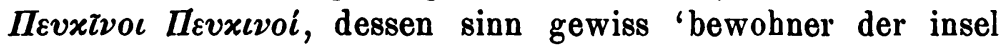
Peuke' ist, steht es, da der name dieser insel selbst sicher ein fremder ist, bei weitem nicht fest, ob wir es dabei mit einer germanischen wortbildung zu tun haben. Einzig die $X \alpha \iota \delta \varepsilon \imath v o i$ bei Ptolemaeus 2, 11,16, aisl. Heiðnir Heinir sind zweifellos bewohner der Heiðmork oder Heiðr und mussen diesen namen, wie sein $d$ gegen $b$ in got. haibi zeigt, sogar schon vor durchführung des germ. accentes erhalten haben. Ganz sicher ist aber der fall auch nicht, denn Heiðnir Heinir und *Haidīnōz

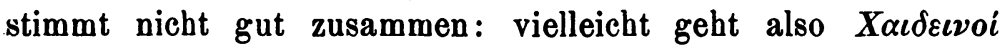
zunächst auf lat. Chaedini zurtick, das selbst wider an stelle von Chaednii getreten sein könnte, geradeso wie Dulgubini bei Tacitus an stelle von Dulgubnii. - Wenn aber gegentuber diesem zweifelhaften und jedenfalls vereinzelten falle zahlreiche germ. adjective auf $-\bar{\imath} n$ und -alīn moralische bedeutung zeigen, so wird man auch in gleichgebildeten germanischen volksnamen zunächst eine solche in anschlag bringen mussen. Und sind wir nicht etwa irre gegangen, wenn wir oben s. 136 in $* S \bar{\imath}$. donez den sinn 'die friedfertigen' vermuteten, so durfen wir auch * Sīdīnōz ebenso verstehen. * Faradīnōz * Farudīnōz (odel * Faratīnōz * Farulīnozz) aber kann leicht 'die reisigen, kriegerischen' oder 'die reisläufer' bedeuten: nach was fur fahrten sollten sie auch sonst benannt sein als nach raub- oder kriegsfahrten?

Schwierigkeiten bereitet uns auch der name Lemovii bei Tacitus und zwar zumal seines suffixes wegen, das in dieser form nicht wohl germanisch sein kann. Ein ausgang -evii oder, was aufs selbe hinauskommt, -ivii, wäre dagegen nicht befremdlich; man vgl. Gambrivii und Hilleviones; denn auch letzteres ist wohl nur ein schwachformiges adjectiv, germ. *illewjonez, das sich zu aisl. illr 'schlecht' verhält wie Gam- 
brivii zu ahd. gambar: der gesammtname für die skadinavischen Germanen stellt sich damit als spottname den namen Lugii, Vandali, Suebi unmittelbar an die seite. Ihren ausgang nimmt die ableitung -ewja- (-iwja-) vermutlich von $u$-stämmen; man vgl. die griech. movierung $\gamma \lambda v x \varepsilon \tilde{\tau} \alpha * \gamma \lambda v x \varepsilon \dot{f} \iota \alpha$ zu $\gamma \lambda v x v_{\text {c. }}$ Daneben kounten aus anderen formen des ursprünglichen paradigmas der adjectivischen $u$-stämme, etwa einem nom. acc. plur. gen. neutr. auf -ewō auch formen wie got. lasiws 'schwach', also $w$-ableitungen mit mittelvocal $e$ ohne secundäre $j a$-ableitung entspringen. Deshalb brauchen ubrigens nicht alle derartigen bildungen wirklich auf $u$-stämme zurlickzugehen, da das suffix innerhalb gewisser bedeutungskategorien productiv geworden sein kann. Viel zahlreicher als im germanischen sind verwante ableitungen in keltischen, wo indessen -euio- durch -ouio- vertreten wird, geradeso wie uns hier neuio- 'neu' in der regel in jügerer gestalt als nouio- begegnet: vgl. Brugmann, Grundr. 1,57. Ist also auch Lemovii in seinem suffixe gallischen namen wie Lexovii, Segovii u. a.m. (Zeuss-Ebel GC. 784) angeglichen und sollte es eigentlich Lemevii lauten? Wenn germanische compositionsglieder in eigennamen wie Habu-, $B a j a-,-r \bar{k} k$ in lateinischer umschrift durch gallisches Catu-, Boio-, -rīg- ersetzt wurden, warum nicht auch einmal eine germanische ableitung durch die ihr entsprechende und geläufigere gallische? Was die wurzelsilbe betrifft, sei an kilâmo 'frequenter' Pa. 190, 23 und manlâmi 'menschlichkeit' N. Bo. $102^{\text {b }}$ erinnert, aus denen Kögel, Zs. fda. 33, 24 mit recht auf eine ablautende nebenform zu ahd. -luomi geschlossen bat. Aus einem worte, das 'frequens' bedeutete, ist ein volksname allerdings noch nicht gut zu verstehen; der in einer reihe von zusammensetzungen wie ags. andlôman 'utensilia, vasa', ags. zelóman 'suppellex, instrumenta', ags. zelóme, ahd. giluomo 'frequenter', ahd. gastluomi 'gastlich', scatuluomi 'schattig', suhtluomi 'pestilens' u. a. m. erbaltene germ. adjectirstamm * lōmu-, * lōmja- ist aber vielleicht von haus aus derselbe wie in mhd. lüeme 'matt, schlaff, mild'. Denn von dem begriffe 'milde' kann die bedeutung 'nicht kärglich gebend, nicht kärglich vorhanden' zu der in obigen zusammensetzungen entwickelten hinuberfuhren: s. Schmeller BW. 1, 1473. Ist aber mhd. lüeme, das mit lahm verwant ist und ursprunglich 'gliederschwach' 
zu bedeuten scheint (s. Kluge EW.4 198 unter lahm), von ahd. -luomi zu trennen, so kann immer noch der volksname damit zusammengehören, wenngleich wir uns dann auf kilâmo und manlâmi als auf belege für die $\bar{x}$-stufe der gleichen wurzel nicht mehr berufen durften. Was entschieden fur zusammenhang mit lahm und lüeme spricht, ist der umstand, dass *lēemenjaz, was die bedeutung anbelangt, ein genaues gegenstuck zu *gambrenjaz, junger *gambrinjaz wäre, woraus sich das gleiche suffix in beiden fällen am besten erklären würde. Sollte $L e-$ movii etwa aus Lomevii verderbt sein, so bleibt natülich die deutung aus mhd. lüeme umsomehr bestehen.

Ist es nun ein zufall, dass auch der name Turcilingi, dessen

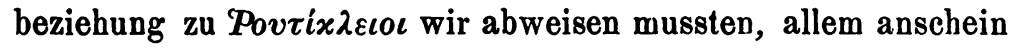
nach etwas ähnliches wie 'schwächlinge' bedeutet? Mit ahd. zorht 'splendidus', wie Zeuss s. 155 wollte, kann er doch kaum zusammenhängen, da er sonst nicht einstimmig in allen quellen, auch bei Jordanes, der den vandalischen königsnamen *Wisumarhs durch Uisumar widergibt, mit $c$ oder $x$ geschrieben sein könnte; wohl aber wird das germ. adj. *turkilaz, das ihm zu grunde liegt, bis auf den unwesentlichen suffixablaut dasselbe sein, wie das, das aus ahd. zorkolôn (Otfr. 3, 23. 25) 'schwer erkrankt darniederliegen' erschlossen werden kann. Freilich sind uns spottnamen von völkern mit dem sinne 'die weichlinge, die schwächlinge, die schwindslichtigen' schon zahlreich genug und in den verschiedensten gegenden Germaniens aufgestossen und etliche werden uns im folgenden noch begegnen. Ihr häufiges vorkommen erklärt sich gerade aus dem hohen ansehen, in dem körperliche kraft und tuchtigkeit bei den alten Deutschen standen. Es könnte sich also auch in dem vorliegenden falle widerum um von einander unabhängige benennungen handeln. Aber dass die nachmals in gesellschaft der Rugen auftretenden Turkilingen deren alte nachbarn, die Lemovier, sind, ist an und fur sich schon nicht unwahrscheinlich. Wer sollten sie sonst auch sein? Und mulsste es nicht befremden, wenn die germanischen Ostseeanwohner zwischen Rugen und Sachsen sich schon vor der völkerwanderungszeit spurlos verloren bätten?

Dass Rugen und Lemovier keine Goten sind, ist bereits oben s. 179 bemerkt worden, ebenso, dass es an beweisen für 
ihre zugehörigkeit zu den Vandilen durchaus gebricht. Mit mindestens ebensoviel recht wie zu diesen, durfen wir sie zu den Ingvaeonen zählen, zumal Plinius die Teutonen (unter deren fälschlich verwendetem namen er die küstenvölker der Ostsee bis zur Weichsel versteht, da sie an anderer stelle bei ihm angeblich nach Pytheas als nachbarn der Goten auftreten) als einen teil der Ingvaeonen aufführt. Oder tut er dies nur, weil die Kimbern Ingvaeonen waren und er die Teutonen zur gesellschaft der Kimbern rechnet? Wenn die Rugen und Lemovier bei Tacitus nicht einen teil der Nerthusvölker, sondern eine gruppe für sich bilden, so ist das kein grund gegen ibre zugehörigkeit zu den Ingvaeonen im allgemeinen; denn die Nerthusvölker bilden doch innerhalb dieser einen engeren kreis, aus dem einige stämme bereits herausgetreten sind: auch die Chauken, die Plinius den Ingvaeonen beizählt, rechnet ja Tacitus nicht zu den Nerthusvölkern. Und ebenso besteht der cultverband, dessen mittelpunkt der Semnonenhain war, zu Tacitus' zeit nur noch aus Sveben, nicht auch aus Chatten und Cherusken, die doch nach Plinius auch Erminonen waren. Rugen und Lemovier durften bereits ihr besonderes stammheiligtum der erdmutter gehabt haben und sie gerade mögen jene pars Sueborum sein, bei der nach Tacitus, Germ. 9 die Isis verehrt wurde, $d . i$. die Nerthus in einer äusserlich abweichenden auffassung: vgl. Zs. fda. 35, 327, wo nur noch ungerechtfertigter weise dem namen Vandilen die herkömmliche falsche ausdehnung gegeben wird.

Ueber vermutungen kommen wir bei all dem freilich nicht hinaus; und ebenso bleiben wir uber die äussere abgrenzung der Ingraeonen nach westen noch sehr im unklaren. Dass die Angrivarier, Ampsivarier, Chasuarier und Friesen zu ihnen gehören, kann bei jedem dieser stämme weder mit bestimmtheit behauptet noch in abrede gestellt werden.

Viel besser sind wir über den bereich des cultverbandes der Nerthus unterrichtet oder doch an der hand der quellen uns zu unterrichten in der lage; und ein gleiches gilt bezliglich der stellung der einzelnen stämme, die ihn bildeten.

Wenn Tacitus, Germ. 40, nachdem er von den Langobarden gesprochen hat, fortfährt: Reudigni deinde et Aviones et Anglii 
et Varini et Eudoses et Suardones et Nuithones fluminibus aut silvis muniuntur, so weist schon das deinde darauf hin, dass hier bei der aufzäblung eine gewisse ordnung eingehalten ist. Die Reudigni sind darum als nordnachbarn der Langobarden zu denken und somit dasselbe volk wie die $\Sigma \alpha \dot{\xi} \xi o v \varepsilon s$ des Ptolemaeus, die sich nach dessen zeugnis $(2,11,7)$ vom " $A \lambda \beta \iota$ bis

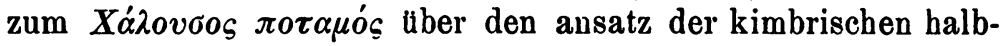
insel ( Reudigni, von dessen endung dasselbe gilt wie von Marsigni (s. oben s. 111), lässt auf germ. * Reudingōz schliessen und hat mit ried, ahd. riot, älter hriot, alts. hreod, ags. hréod schon des anlautes wegen nichts zu tun. Mit recht hat man dagegen

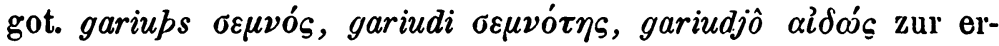
klärung beigezogen; aber freilich war es verfehlt, diesen worten

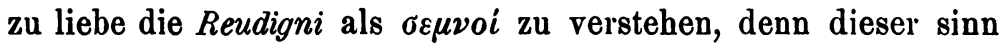
kommt erst durch zusammensetzung mit $g a-z u$ stande: gariups bezeichnet einen, der öfter rot wird, und daher einen schamhaften, ehrbaren; beim volksnamen dagegen haben wir uns an das einfache germ. * reudaz zu halten. Dieses ist eine ablautform von rot, germ. * raudaz, scheint aber eine eigentümliche bedeutung ausgebildet zu haben: denn wenn der Gote den begriff $\sigma \varepsilon \mu \nu o ́ s$ durch gariups, nicht durch *garaups ausdrückte, muss ihm * riups von der röte des gesichtes gegolten haben, und ebenso ist aisl. rjöðr soviel als 'ruddy' (s. Cleasby-Vigfússon 500); man beachte besonders den ausdruck rauðan ok rjóðan, der Rígsmál 21 von Karl, dem sohne des Afi und der Amma, gebraucht wird, wobei das erste beiwort auf die farbe des haares, das andere auf die des gesichtes, zumal der wangen, sich beziebt und das bluhende aussehen kennzeichnen soll. Reudigni * Reudingōz wird man demnach als 'leute von rötlicher, bluhender gesichtsfarbe' zu verstehen haben.

Einen anderen alten namen der Sachsen bewahrt uns das Widsiolied. Und zwar ist dies der name Myrzinzas, den man bisher irrtümlich mit dem der Maurungen zusammengebracht und für den einer svebischen abteilung gehalten hat. Dazu hat freilich das Widsiolied selbst anlass gegeben. Denn wenu es unmittelbar, nachdem rom kampfe der Enzle mit den Myrzinzas und der festsetzung der grenze zwischen beiden die rede war, im v. $43 \mathrm{f}$. mit beziehung auf diese grenze heisst: héoldon 
forð̀ sippan Enzle and Swáfe swá hit Offa zeslóz, so scheint die gleichsetzung der Swcéfe und Myrzinzas gerechtfertigt, ja sogar geboten. Allein Saxo Grammaticus (ed. P. E. Muller 1, $170 \mathrm{ff}$.) berichtet uns von einem kampfe des Uffo mit den Sachsen, der unbestritten derselbe ist wie derjenige mit den Myrzinzas, auf den das Widsiolied anspielt und im selben locale ausgetragen wird: wie im ags. liede Fifeldor, so wird bei Saxo die Eider (fluvius Eidorus) genannt, was ganz auf das gleiche hinauskommt. Die Sachsen können aber unmöglich als Sveben bezeichnet werden. Auf der einen oder anderen seite muss daher ein irrtum vorliegen, und auf welcher dies der fall ist, kann auch sofort als ausgemacht gelten, da an der Eider niemals Sveben, wohl aber Sachsen nachbarn der Angeln waren und mit ihnen krieg führen konnten. Die Sachsen sind zudem im ersten katalog des Widsio gar nicht genannt, was doch sehr auffallen müsste, wenn sie sich nicht hinter den im v. 22 aufgefuhrten Myrzinzas verbergen. Wenn es ferner v. 22 f. heisst: Witta wéold Swáfum, Wada Hálsinzum (Holsinzum?), Méaca Myrzinzum, Mearchealf Hundinzum, so können Swáfe und Myrzinzas nicht dasselbe sein, es sei denn, dass von diesen beiden benachbarten versen der eine unecht ist.

Enzle and Swáfe im v. 44 muss nun notwendig in Enzle and Seaxan gebessert werden. Die verwechslung der Sachsen und Schwaben ist nicht allzu auffällig, wenn sonst im germanischen epos Eruler und Hearobearden, Langobarden und Goten rerwechselt werden. Noch dazu liegt bei diesen seitenstucken der grund des irrtums bei weitem nicht so klar zu tage als in unserem falle. V. 61 lautet nämlich: Mid Englum ic was and mit Swcéfum and mit Enenum; und waren hier Angeln und Schwaben in stehender verbindung, so konnte diese, so lange das lied mundlich uberliefert wurde, leicht auch im v. 44 das ursprüngliche Enzle and Seaxan verdrängen.

Myrzinzas und Mauringa unter einen hut zu bringen, werden wir uns nun nicht mehr bemuhen. Ohnedies hat alle anstrengung in dieser richtung bisher nichts gefruchtet. Man sieht nicht ein, was es helfen soll, wenn man mit Mullenhoff s. 97. 99 Mŷrgingaland und Mŷrging schreibt: das $g$ bleibt dabei immer noch unerklärlich. Auch von einem grammatischen wechsel von $w$ aus $g w$ vor -ing fur die starken casus mit $g$ vor -ung

Beiträge zur geschiohte der deutschen sprache. XVII. 
fur die obliquen, wie Möller will (Ae. volksepos 28), kann hier nicht wohl die rede sein, da das $w$ in marawi, muruwi schon ein vorgermanisches ist, wie die keltischen entsprechungen zeigen: vgl. s. 112. Heinzel, der die unvereinbarkeit von Mauring und Myrzinz richtig erkennt, vermutet deshalb, Ostg. heldensage (WSB. 119 [1889]), 25, dass ags. Miering volksetymologisch zu mirize 'merry' gestellt wurde; und da nunmehr von einem zusammenhange mit Mauring abzusehen ist, wird es in frage kommen, ob Myrzinzas nicht von haus aus als eine weiterbildung aus myrze mirize zu gelten hat. Allein dieses wort weist ebenso wie ahd. murg 'mutabilis', murgfâri 'caducus, fragilis, transitorius' und got. gamaurgjun 'kürzen' auf germ.

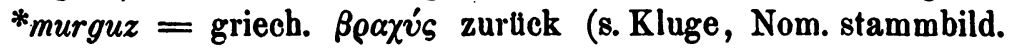
$\S 180$. Noreen, Urg. judl. 7), heisst also ursprunglich 'kurz', dann 'kurzweilig', aber auch im ags. noch nicht 'munter, fröhlich' von personen, geschweige denn in der zeit, da der name Myrzinzas aufgekommen ist. Neben germ. *murguz $\beta \rho \alpha \chi v ́ s ~ s c h e i n t$ es aber noch ein ahnliches, mit lat. marcere, marcescere, marcidus verwantes germ. wort gegeben zu haben. Man vgl. mhd. murc 'morsch, faul' das allerdings nur im nom. (im reim auf burc) belegt ist: 8. Lexer, MW. 1, 2250; vor allem aber mitteldeutsch morgen, morghen 'saft- und kraftlos, schlaff, mager' (Weigand DW. 2, 77). Aisl. morkinn 'putridus' zeigt dagegen andere abstufung des gutturals, die vielleicht aus einer vorgerm. stammform mrknó- zu erklären ist. Man beachte ubrigens die gleichartige ableitung in md. morgen, aisl. morkinn und in norw. mauren 'spred' (Aasen 484).

Es scheint also hier widerum ein namenpaar nach art von

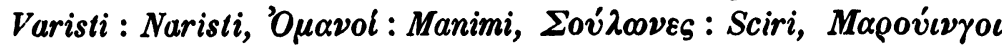
: Juthungi vorzuliegen. $\mathrm{Zu}$ den beiden letztgenannten namen stimmt Myrzinzas : Reudigni zudem in der bedeutung: wie jene 'die schlaffen' und 'die jugendfrischen', sind diese 'die saftund kraftlosen' und 'die mit bltuhender gesichtsfarbe'. Vermutlich ist der spottname den Sachsen von ibren mit ihnen verfeindeten nordnachbarn, den Angeln, aufgebracht, jedenfalls aber viel länger festgehalten worden, als man ihn in seinem ursprttnglichen sinne verstand. Wenn bei Saxo (ed. P. E. Muller 1, 387) dem könige Sigurd Ring die worte in den mund gelegt sind: ex parte adversa perpaucos Danos esse, Saxones com- 
plures aliasque effoeminatas gentes in acie stare, so ist hierbei der vorwurf gegen die Sachsen nicht so zugespitzt, dass man ihn mit einem geläufigen auf sie gemulnzten scheltworte in verbindung bringen durfte. Die stelle ist aber mit ein zeugnis dafur, wie gerne gerade völker, denen kriegstluchtigkeit als das höchste galt, ihren gegnern diese abstritten; vgl. auch Saxo p. 300.303.

Von dem festen grund, den wir bei den Reudigni des Tacitus bereits gewnnnen haben, ist es nun ein leichtes weiter fortzubauen. Zunächst folgen diesen in seinem verzeichnisse die Aviones und dann gleich die Anglii. Da wir aber die Angeln bereits aus dem Widsið als die nordnachbarn der Sachsen auf dem festlande kennen gelernt baben, kann es uns nur willkommen sein, wenn uns der wortsinn des namens der Aviones gestattet, ja sogar nötigt, mit ihnen auf die inseln hinauszurlicken. Denn klärlich sind die Aviones germanische *Anjonez, gotische *Aujans 'inselbewohner': vgl. J. Grimm GDS. 472. Es fragt sich nur, ob sie nach westen oder osten, ob sie auf die nordfriesischen oder dänischen inseln gehören.

Die ersteren kommen wegen ihres geringen umfanges als bereich eines besonderen volksstammes von vornherein schon weniger in betracht, und man wird sich daber lieber fur die anderen entscheiden; zuversichtlich aber dann, wenn gezeigt werden kann, dass sie noch zum gebiete der Nerthusvölker gehören, und dass uns ein anderer volksname, den wir auf sie ansetzen könnten, von Tacitus nicht zur verfugung gestellt wird. Macht doch dessen verzeichnis der Nerthusvölker durchaus den eindruck der vollständigkeit.

Die frage, die wir uns hier gestellt haben, hăngt aufs innigste zusammen mit der nach der lage der Nerthusinsel selbst. Wenn wir auch diese hiermit neuerdings aufwerfen, soll damit nicht gesagt sein, dass sie nicht schon da und dort richtig beantwortet wurde; aber, wo dies der fall war, geschah es nur ganz zufällig und ohne dass man die wirklich stich. haltigen gründe vorgebracht hätte. Und doch liegen diese nahe genug.

Wenn es heute nicht mehr möglich ist oder nur durch funde ermöglicht werden könnte, die stelle des svebischen oder lugischen stammheiligtumes zu ermitteln, so liegt der haupt- 
grund hierfur darin, dass das eine wie das andere in einer gegend lag, die von Germanen in der völkerwanderungszeit verlassen wurde und an ibrer statt slavischen einwanderern zufiel. Im bereiche der Nerthusvölker dagegen trat lediglich ein germanischer stamm an stelle von anderen, von denen uberdies reste genug mit der neueingedrungenen nordischen bevölkerung verschmolzen, um dieser die alten uberlieferungen zu vermitteln. Auch erhielt sich das heidentum hier so lange, dass wir ther seine formen und seine cultstătten gut unterrichtet sind.

Von vornherein aber und aus ganz allgemeinen gründen ist es die insel Seeland, die hier unsere aufmerksamkeit auf sich lenkt. Denn wo sollten sich die Germanen die erntesegen spendende erdmutter besonders heimisch gedacht haben, als in dem fruchtbarsten lande, das sie von haus aus besassen? Das ist aber unbestreitbar Seeland. Mit recht wird diese insel von Adam von Bremen in der Descriptio insularum aquilonis 7, 370 als opulentia frugum celeberrima und von Saxo in der vorrede seines geschichtswerkes (ed. P. E. Muller 1, 11) als conspicua necessariarum rerum ubertate laudanda und amoenitate cunctas nostrae regionis provincias antecedens gerthmt: sie ist auch heutzutage weitaus die bluhendste provinz des dänischen staates.

Mit dieser naturlichen beschaffenheit des landes wird es wohl zusammenhängen, wenn von der sage Seeland als der eigenste erwerb der göttin Gefjon hingestellt wird, in der wir Zs. fda. 35, 327 bereits die Nerthus erkannt haben. Freilich hat Mullenhoff s. 362 den schauplatz der ganzen sage von Gefjon und Gylfi nach Schweden verlegen wollen, wegen der angeblich nur aus einer falschen etymologie herstammenden verbindung Seelands (das als 'seeland' gefasst wurde, wogegen es ursprünglich Selund d. i. 'die an seehunden reiche insel' hiess: s. Bugge, Arkiv 6, $237 \mathrm{ff}$.), mit dem Mälarsee. Doch findet sich eine anspielung auf die sage schon in einer Bragi dem alten zugeschriebenen strophe, die dann falsch und diesem erst sehr spăt untergeschoben sein mutsste, da zu seiner zeit der name der insel noch nirgends volksetymologisch entstellt war. Zudem bedeutete sár im aisl. gar nicht und auch im altdän. nicht in erster reihe 'see' in unserem sinn, d. i. 'stehendes binnen- 
gewässer'. Aber wenn man Mullenhoff schon zugeben wollte, dass die verbindung Seelands mit dem Mälarsee nur die folge einer volksetymologie sei, so ist damit noch gar nicht ausgemacht, ob der see oder ob die insel der sage ursprunglich angehört. Es würde vielmehr erst darauf ankommen, welche vorstellung naturlicher erscheint, die des lospflugens einer insel vom gegenuberliegenden lande, von dem sie nur durch einen sund getrennt ist, oder die von Mullenhoff vorausgesetzte des herauspflugens eines gar nicht natülich begrenzten landstriches aus einem see, noch dazu einem rings von felsigen ufern eingeschlossenen und mit inseln ubersäten, wie es der Mälar ist. Freilich konnte, wenn die ursprungliche gestalt der sage die war, dass Seeland durch Gefjon von Schonen abgepflugt wurde, das land nicht von haus aus als einem Schwedenkönig Gylfi abgenommen und nicht als dem Dänenreiche erst zugefügt erscheinen. Aber hier kann leicht eine verschiebung stattgefunden haben, indem an die stelle der Ingraeonen in der herschaft uber Seeland und damit auch in der sage, die gleichfalls ursprunglich ingvaeonisch gewesen sein wird, die Dänen traten: notwendig musste dann, weil Schonen auch dänisch war, der könig, dem Gefjon das land abnimmt, nach Schweden hinausgertickt werden. Auf diese art erklärt sich sogar ganz einfach und ungezwungen die gewiss auffällige und anstoss gebende verbindung der insel mit einem so ferne liegenden local. Eine ubertragung Gylfis von einem nichtschwedischen volke auf die Schweden muss doch auch Mullenhoff annehmen, ohne dass er indes einen grund für sie anzugeben weiss.

Wenn uns in der Ynglingasaga c. 5 erzählt wird, dass Oðinn die Gefjon dem Skjoldr, dem ersten könige von Dänemark, vermählt habe, so haben wir allein deshalb, weil die ubrigen quellen dieses umstandes geschweigen, auch noch nicht grund genug, dabei an eine erfindung des Snorri zu denken. Da vielmehr Skjoldr, der in der Fms. 5, 239 als Skánunga goð bezeugt ist, in den ags. genealogien als Sceldwa oder Scyld mitten unter Frey- und Njorðhypostasen seinen platz hat und an der spitze der Hleiðrkönige ebenfalls stammvater eines Freygeschlechtes ist, nur Njorðr selbst sein kann, und andrerseits Gefjon die Nerthus ist, so tritt uns hier deutlich das eheverbundene göttliche geschwisterpaar und zwar in beziehnung zu 
Seeland und den Hleiðrkönigen entgegen. Dass diese so vielfach vertreter von Vanengöttern sind - man denke nur an die verschiedenen Fróði - weist schon darauf hin, dass vor allem den Vanen zu ehren in Hleiðr (Lederun) jene grossen opferfeste gefeiert wurden, von denen noch Thietmar von Merseburg 1, 9 berichtet: sie sind auch gar nichts anderes als das Fröblod, das nach Saxo (ed. P. E. Muller 1, 50) von Haddingus, selbst einem deutlichen vertreter des Njorðr, eingesetzt wurde. All das lăsst uns Hleiðr als eine alte und zwar als die im ganzen bereich der Dänenherschaft weitaus bedeutendste Vanencultstätte erkennen. Und da der ort auf einer meeresinsel - auf Seeland - gelegen ist, ja sogar der see in seiner nächsten nähe nicht fehlt - es ist der Videsø bei Ledreborg, - so ist nicht zu zweifeln, dass wir dort an der stelle des ingvaeonischen Nerthustempels stehen. Ja auch der name Hleiðr, der als appellativum im nordischen nicht belegt, aus dem ablautenden got. hleipra $\sigma x \eta v \eta \dot{~ j e d o c h ~ i n ~ s e i n e m ~ s i n n e ~}$ verständlich ist, stammt wohl noch aus vordänischer zeit und erhält uns die ingvaeonische bezeichnung für das gebäude, in dem wagen und bild der göttin verwahrt wurden.

Dem ansatz der taciteischen Avionen auf den dänischen inseln steht also nichts mehr im wege. Wir werden aber sogleich widerum fragen, wie sich Ptolemaeus zu ihnen verhält.

Wir finden bei diesem gewährsmanne als nordnachbarn

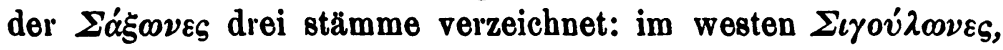
weiter östlich $\Sigma \alpha \beta a \lambda l \gamma \gamma \iota \iota$, am östlichsten Koßavdoi. Es ist aber recht befremdlich, dass die kimbrische halbinsel gerade dort, wo sie am schmălsten ist, drei völkern neben einander raum gewăhrt baben soll. Andrerseits spricht Ptolemaeus von drei kleineren Skandien, d. i. Seeland, Funen und LaalandFalster, die indessen auf seiner karte auf einen bruchteil ihres umfanges eingeschrumpft sind; und vielleicht hängt es damit zusammen, dass er aus ibrem bereich keinen volksnamen uberliefert. Aber sollte ihm oder seinem gewăhrsmanne von dort in wahrheit keiner zugekommen sein? Setzte er namen die auf die inseln gehört hätten auf das festland, so machte er sich nur desselben fehlers schuldig wie Tacitus, der von seinen sieben Nerthusvölkern, die inselbewohnenden Aviones eingeschlossen, Germ. 40 sagt: fluminibus aut silvis muniuntur. Wenn 
wir bei beiden dieselbe folge in der aufzählung voraussetzen, so

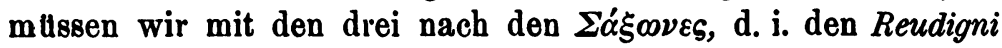
des Tacitus, aufgefubrten namen gegen osten ins meer hinausrucken: ihre dreizahl entspricht dann nicht nur den drei Skandien des Ptolemaeus sondern auch der tatsächlichen gliederung des landes. Aviones bei Tacitus dagegen ist der alle drei stämme umfassende gesammtname.

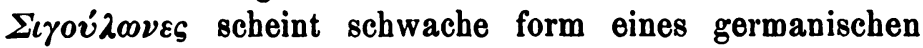
adjectivs * sigulaz zu sein, das wie got. sakuls zu sakan, aisl. gjofull zu gefa, ahd. ezzal zu ezzan (8. Kluge, Nom. stammbild. § 192) zu ahd. alts. sìgan, ags. sizan, aisl. siga gehören wird. Abgesehen von der hier. nicht verwendbaren ursprunglichen bedeutung des wortes heisst ndl. zijgen auch 'hinfallen, in ohnmacht fallen', ebenso mhd. seiger nicht nur 'langsam tröpfelnd', sondern auch 'matt, marcidus', ahd. seiglîh 'languidulus'; norw. sigin 'sunken, lidt falden eller sammentrykt' und daneben 'træt, mødig, udmattet' (Aasen 643).

Ziehen wir die ubrigen germanischen volksnamen zum

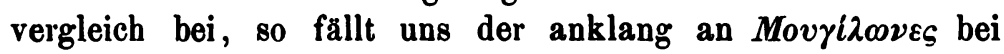
Strabo p. 290 auf, und dieser ist um so merkwürdiger, als sich uns für *Sigulonez eben die bedeutung 'die hinfälligen, ohnmächtigen', fur *Mūgilonez fruber (8.51) gerade die gegenteilige, nämlich 'die mächtigen, starken' als wahrscheinlich ergeben hat. Sollte hier etwa ein ähnliches wortspiel vorliegen wie bei Varisti. Naristi, durch beide namen also derselbe stamm bezeichnet werden? Bestätigte sich das, so wurde damit unsere vermutung ther die weite erstreckung des machtbereiches des Maroboduus - denn zu seinem anbange werden die Movyi2

Und wirklich lässt sich auch $\Sigma \alpha \beta \alpha \lambda l \gamma \gamma \iota 0 \iota$ bei Ptolemaeus mit $\Sigma \iota \beta \iota v o i$ bei Strabo vereinigen, zu dem es sich etwa so verhalten kann wie Sibeke zu Savulo Seafola Sabene. $\Sigma \alpha \beta \alpha$ $\lambda i$ ryı int-seffen, alts. $a f-$, an-sebbjan = lat. sapere) gebildetes adj. *sabalaz 'verständig, scharfsinnig' voraus (vgl. Mullenhoff, Zs. fda 6,459 ), aus dem dann mit dem so vielfach verwendeten suffix -inga- eine substantivierung erfolgen konnte. Dass der rolksname patronymischen charakter habe, wie Henning, Runendenkmäler 124 vermutet, scheint mir eine ferner liegende mög- 
lichkeit zu sein, die sich gleichwohl nicht ganz abweisen lässt. Das ` der ableitung ist wohl zu streichen; oder soll es eine zwischen gemeingerm. -inga- und nord. -ingjan- (Kluge, Nom. §27) mitten inne stehende gleichbedeutende ableitung -ingjagegeben haben? $\Sigma i \beta \iota v o l$ dagegen kann zu einem germ. adjectiv * sebīnaz (got. * sibeins) gehören, das $\mathrm{zu} * \operatorname{seb} \bar{o}(n)$, alts. sebo, ags. sefa, aisl. sefi ebenso gebildet wäre wie etwa alts. nîঠin 'feindselig' zu nîঠ; vgl. Kluge, Nom. stammbild. § 199. Der einwand, dass es bei Strabo dann $\Sigma \varepsilon \beta \iota v o i$ heissen mlisste, ist nicht stichhaltig, da wir auch schon bei Velleius Paterculus

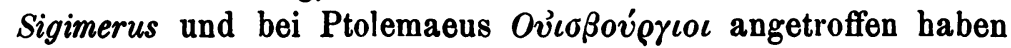
(vgl. oben s. 133).

So wie statt $\Sigma \alpha \beta \alpha \lambda i \gamma \gamma \iota \iota \iota \Sigma \alpha \beta \alpha \lambda i \gamma \gamma o \iota$, so erwarten wir

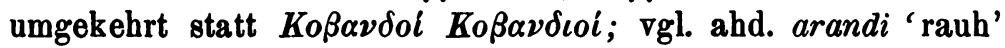
und barranti 'starr' (Kluge, Nom. stammbild. § 235). . Eine verderbnis wäre aber hier sehr leicht aus dem Cubandi $=$ Cubandii einer lat. vorlage zu erklären. Was die wurzel betrifft, darf man vielleicht an norw. kubbe (und kubb) m. 'klods, blok, kort stump af en træstamme' (= isl. kubbi, kubbr, schwed. kubb, norw. kubben (kubbut, kubbet) 'but, stump, rundagtig'), noget tyk og kort' erinnern, denn germ. *kubandjaz 'stumpf, kurz' wurde derselben bedeutungskategorie wie ahd. arandi, barranti angehören. Und widerum lässt sich auch Bov́roveṣ bei Strabo, wie es uberliefert ist, in einem ganz ähnlichen sinne verstehen: isl. butr und kubbi, kubbr, norw. butt und kubbe, kubb ist durchaus gleichbedeutend und ndl. bot, ndd. but, dăn. but, span. boto, franz. bot in pied-hot 'klumpfuss' soviel als 'kurz, stumpf, plump'.

Als die östlichsten in der den taciteischen Aviones entsprechenden völkergruppe bei Ptolemaeus sind die Koßavdoi auf Seeland anzusetzen, also dort, wo uns nachmals Eruler begegnen.

Die hindeutung auf die wobnsitze dieses stammes bei Mamertinus Paneg. Maximiniano Aug. dictus (a. 289) c. 5 (viribus primi, locis ultimi barbarorum) und ebenso die bei Sidonius Apollinaris in seiner epistel aus Burdegala 8, 9 (Hic glaucis Herulus genis vagatur, Imos oceani colens recessus, Algoso prope concolor profundo) wäre allerdings auch $\mathrm{zu}$ verstehen, wenn sie vom suld- oder nordufer der ostsee kamen; dass aber das 
nicht der fall ist, ergibt sich aus der nachricht des Jordanes c. 3, dass die Dänen (die ursprunglich in Schonen sassen) die Eruler aus ihren stammsitzen vertrieben hätten: Dani ex ipsorum (Scandzae cultorum) stirpe progressi, *qui Herulos propriis sedibus expulerunt. Das alte Erulerland muss darnach einen teil des späteren Dänemark ausmachen. Ueberdies hat Müllenhoff, Beovulf $31 \mathrm{ff}$. durch eine lichtvolle untersuchung gezeigt, dass auch noch die dänische königssage, vor allem im Beowulf und Widsio, die erinnerung an siegreiche kămpfe der Dänen mit Erulern auf Seeland bewahrt, wenn auch die letzteren nicht mehr unter diesem namen bekannt sind. Selbst die zeit, in die ihre austreibung fällt, lässt sich einigermassen bestimmen, wie dies ebenfalls schon Müllenhoff, Beovulf $30 \mathrm{f}$. versucht hat. Denn im jahre 459 und zuletzt unter der regierung des westgotischen königs Eurik werden noch Eruler aus der Ostsee erwähnt (s. Zeuss s. 479), im jahre 512 aber, als ein teil der von den Langobarden besiegten Donau-Eruler nach norden zurtickwandert, sitzen sogar am Kattegat bereits Dänen und ein selbständiges Erulerreich im norden besteht damals nicht mehr, wie aus den berichte Prokops uber jene rückwanderung, De b. Goth. 2, 15 (s. Zeuss s. 481) deutlich hervorgeht. - Darnach ist es, wie ich beiläufig bemerken will, gar nicht ausgeschlossen, dass erulische runendenkmäler auf uns gekommen sind, und es verdient gewiss beachtung, dass das Hariso der spange von Himlingøje auf Seeland sonst als germanischer name nicht belegt ist, ausser gerade als erulischer auf einer inschrift, CIL. 5, 8750.

Wenn im Widsio und Beowulf die Eruler unter dem namen der Heaðobearden auftreten, so wird dieser irgendwie von den Barden im Bardengau, den Bardi bellicosissimi Helmolds, auf sie ubertragen worden sein. Werden sie nebenbei Widsio 47 Wicinza cynn genannt, so lässt dies allerdings noch einen zweifel zu, ob dabei ein anderer eigentlicher volksname vorliegt. Wenn es aber v. 59 heisst: Mid Wenlum ic waes and mid Warnum and mid Wicinzum, so wird es schon (trotz Mullenhoff, Beovulf 97) völlig klar, dass Wicinzas in der tat ein ags. name fur die Eruler gewesen sein muss. Und zwar stimmt derselbe sebr wohl zu der tatsache, dass diese vom ende des 3. bis in die zweite hälfte des 5. jahrhunderts hinein als äusserst verwegene 
seeräuber die klisten des Römerreiches verheeren: vgl. Zeuss s. $477 \mathrm{ff}$. Vielleicht hat gar zur ausbildung des begriffes wiking der umstand mit beigetragen, dass das volk der Wikinge ein seeräubervolk $x \alpha \tau^{\prime}{ }^{\prime} \xi \chi^{\prime} \dot{\eta}^{\prime} \nu$ war. Germ. minkingaz scheint von haus aus weitere bedeutung gehabt zu haben. Wenn Mullenhoff Beovulf 96 (Bugge, Studien 1881 s. 6 folgend) der meinung ist, dass die Angelsachsen die bei ihnen einbrechenden und in festen lagern sich einnistenden nordleute nach ags. wic als wicinzas 'lagerer' bezeichnet hätten, so widerspricht dem schon die verwendung des wortes als name für die Eruler; ausserdem war das aufschlagen von lagern doch nichts fur die nordischen seeräuber im gegensatze zu anderen, die in fremdem lande krieg fubrten, charakteristisches. Aber die herleitung von wic kann man gelten lassen: * ${ }_{\text {wikingaz }}$ wird ursprlunglich derjenige sein, der sich im lager - im gegensatze zum hause - aufzuhalten pflegt, der also den krieg als beruf gewählt hat; man vgl. die worte, die Caesar BG. 1, 36 dem Ariovist in den mund legt: intelleclurum, quid invicti Germani, exercitatissimi in armis, qui inter annos XIIII teclum non subissent, virtute possent; ferner Ynglingasaga 34: váru margir sákonungar peir er réठu liði miklu, ok áttu engi lond; bótti sá einn meঠ fullu heita mega sákonungr, er hann svaf aldri undir sótkum ási, ok drakk aldri at arinshorni. Unter solchen umständen fällt es auf, dass die nachbarn der Eruler-Wikinge, die Dänen mit alterem namen bei Ptolemaeus $\Delta \alpha v x i$ coves heissen, worin wir bereits eine ableitung von germ. *dauka- 'haus' erkannt haben. Es liegt nicht ferne, hier an einen gegensatz der im hause zu den im lager lebenden zu denken. Aber besser noch wird man germ. *Daukjonez als olxetoc in dem sinne 'die freundlichen, die vertrauten' verstehen.

Auch auf die möglichkeit eines anderen gegensatzes zwischen Dänen und Erulern sei noch hingewiesen. Wenn es bei Jordanes c. 3 heisst: Suetidi cogniti in hac gente corpore eminentiores: quamvis et Dani, ex ipsorum (Scandiae cultorum) stirpe progressi Herulos propriis sedibus expulerunt, qui inter omnes Scandiae nationes nomen sibi ob nimia proceritale affectant praecipuum, so erfordert hier der ganze zusammenhang eine verschiebung des qui an die stelle unmittelbar vor Herulos, es sei denn, dass wir einem so schlechten stilisten wie Jordanes eine nicht sinn- 
gemässe ausdrucksweise zumuten; sicherlich wird nur von den Dänen ausgesagt, dass sie sich auf ihre körpergrösse etwas besonderes zu gute taten. Und war das der fall, so konnten sie sehr wohl ihre kleiner gewachsenen nachbarn als 'knirpse' bezeichnen. Nichtsdestoweniger möchte ich auf die frther er-

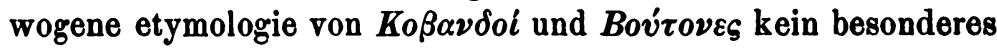
gewicht legen, so lange es an älteren belegen für eine wurzel kub und but 'kurz, stumpf' gebricht. Auch sind die germanischen stammnamen bei Strabo dort, wo wir sicher wissen, um welche es sich handelt, zum teile so entsetzlich verunstaltet, dass wir fur die ubrigen nichts gutes voraussetzen durfen; ja es mlisste uns fast wunder nehmen, wenn uns irgendwo eine ganze gruppe von wohl erhaltenen aufstiesse. Was oben uber

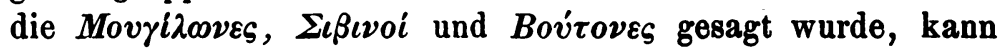
deshalb nicht als melr denn eine möglichkeit gelten, neben der, wie ich denke, als am meisten in betracht kommend diejenige bestehen bleibt, dass der herschenden annahme gemãss Bov́-

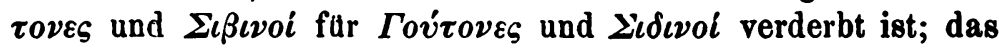
mitteninnestehende Movyíaves brauchte man dann nur in Povyiגoves zu ändern, um an die zwischen Goten und Sidinen sesshaften Rugen anzukntipfen; denn wenn diese *Rugīz und daneben * Rugiklīo hiessen, warum nicht auch mit einem dritten, nur mittels eines anderen deminutivsuffixes gebildeten namen *Rugilonez?

Um nun wider zur kimbrischen halbinsel zurtickzukehren, so mulssen dort die Xádo des Ptolemaeus den Anglii des

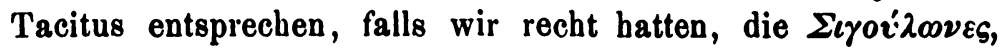
$\Sigma \alpha \beta \alpha \lambda i \gamma \gamma \iota \iota$, Ko $\beta \alpha v \delta o i$ des einen zusammen den Aviones des anderen gleichzusetzen. Sicher sind die Angeln nicht 'bewohner des winkels'; vielleicht aber $\alpha \dot{\gamma} x v \lambda_{0 \mu \tilde{\eta}} \tau o \iota$ und mit dem namen Xádoı vom hehlen als 'die verschlagenen, listigen' bezeichnet: vgl. norw. haal (= aisl. háll, ahd. hali, mhd. hoele), das nicht nur 'glat, slibrig' sondern auch 'slu, listig' bedeutet (Aasen 272) und neben dem gleichbedeutendes schwedisches hal auf eine ablautende nebenform mit kurzem vocal hinzuweisen scheint, da man als entsprechung zu aisl. háll im schwedischen hål erwartet.

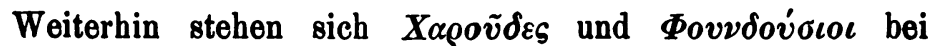
Ptolemaeus und Varini Eudoses bei Tacitus gegentuber, Ersterer 


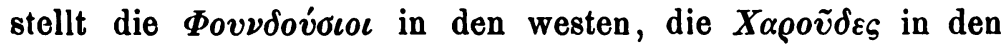
osten. Damit stimmt es freilich nicht, dass das land, das den namen der letzteren fortbewahrt, Hor d á Jóllandi (Fornald. 1, 114), dän. Harthesyssel, jetzt Harsyssel, zwischen dem Limfjord in norden und der Skærnaa im süden, an der westseite der jütischen halbinsel gelegen ist (s. Nielsen, Bidrag til oplysning om sysselinddelingen i Danmark 64. Bugge, Beitr. 12,10). Allein das braucht uns nicht zu beirren, da in Jutland in folge der auswanderung eines teiles seiner ingvaeonischen bewohner und des eindringens der Dänen eine verschiebung der stämme nach westen hin erfolgt sein kann, wie uns eine ähnliche vielfach anderorts begegnet: man denke nur an die lage des gaues Borahtra oder Hattuaria im vergleich zu den alten sitzen der Bruktern und Chattuarier.

Ob die namen Varini und $\boldsymbol{X} \alpha \rho o \tilde{v} \delta \varepsilon \varsigma$ zu einander in irgend welcher bedeutungsbeziehung stchen, ist schwer zu sagen, da sie sich unserem verständnis uberbaupt nicht voll erschliessen. Von dem ersteren lässt sich wenigstens mit einiger sicherheit behaupten, dass er von haus aus eine participialbildung sei zur germ. verbalwurzel war 'sehen, achten auf, sorgen fur', die uns schon im namen Varisti in veränderter bedeutung untergekommen ist. Das später vielfach begegnende Varni Ovóovoı (s. Zeuss $361 \mathrm{ff}$.) zeigt dieselbe ableitung ohne mittelvocal, ist also als germ. *War-noz neben *War-enōz anzusetzen, sofern nicht ein got. plural *Warnôs, gen. *Warnê. mit synkope wie in haipnô 'heidin' (8. Kluge in Pauls Grundr. 1, 381) anlass zu dieser jüngeren schreibung gegeben hat. Was den namen der Haruden betrifft, so stimmt derselbe in seiner bildung auffallend $\mathrm{zu}$ der unseres held. Man vgl. Harudes bei Caesar, Charydes auf dem Mon. Anc., Xaৎoṽ $\delta \varepsilon \varsigma$ bei Ptol., Harudi in den Ann. Fuldens.,

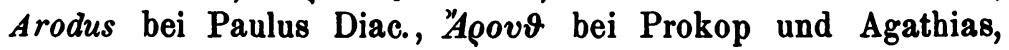
Harud Haruth im Necr. Fuld. (Förstemann DN. 1, 636), Arochi statt Arothi Harothi (s. Mullenhoff s. 66) bei Jordanes, altn. Horðir (auf dem Rökstein noch gen. sing. Harups) mit aisl. holdr; "Aopdos bei Prokop mit aisl. hauldr (Noreen, Urgerm. judl. 44); Hoereðaland in der Sachsenchronik, *Horeðas im Widsið, Haereð im Beowulf und ahd. Harid, Harido (Förstemann DN. 1, 637) mit ags. hoeleðas, ahd. helid, alts. helip. Wegen des wechsels von germ. $d$ und $b$ im suffixe muss dessen dental 
vorgerm. $t$ gewesen sein. Lägen einzig die formen mit mittelvocal $u$ vor, so durfte man unbedenklich verwantschaft mit griech. xov́ $\emptyset \tau \varepsilon \varsigma, K o v \varrho \tilde{\eta} \tau \varepsilon \varsigma$ annehmen. $\mathrm{Da}$ das $\eta$ in diesen worten doch wohl erst aus dem nom. sing. eingedrungen ist, lãsst sich für sie eine grundform $x \circ \rho \mathcal{F} \varepsilon \varepsilon \varsigma$ erschliessen und zu dieser könnte sich germ. *harwupez *harwudez, das lautgesetzlich zu *harupez *harudez werden musste, ebenso verhalten wie aisl. holdr holdr (aus halupr) zu kelt. calet- in Caletes u. a. m. (s. oben s. 15). Griech. xov́ $\eta \tau \varepsilon s$ bedeutet 'junge mannschaft'; das wort wäre also auch vorzugglich geeignet, einen volksnamen

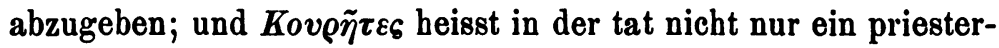
geschlecht des Zeuss auf Kreta, sondern auch ein alter volksstamm in Aetolien. Allein ags. Hoereðas ist aus einer grundform *Koruetes schlechterdings nicht zu erklären. Es bliebe also, wenn man die in betracht gezogene etymologie dennoch aufrecht halten wollte, als einziger ausweg die annahme ubrig, dass die in der tat in alter zeit auch bei den Ingvaeonen nicht belegte namenform mit $e$ der ableitung einer jüngeren angleichung an das ähnliche und bedeutungsverwante hceleðas ihren ursprung verdanke; und das liegt durchaus nicht ausser dem bereich der möglichkeit, wie zahlreiche verwante vorgänge zeigen.

Von dem einklang der namen der norwegischen Hordir und der ingraeonischen Charudes gilt dasselbe, was fruher uber die den Horठir benachbarten Rygir und die sudgermanischen Rugii bemerkt wurde. Wir haben widerum eher an ethnographischen zusammenhang als an eine zufällige gleiche benennung zu denken. Die Harudi der Ann. Fuld. werden wohl ein an den Harz versetzter bruchteil der jutländischen Haruden sein und identisch mit den Warnen der lex Angliorum et Werinorum und des Fredegar c. 15. Wenn ihr name nur antikisierte benennung der 'bewohner des hardtes' wäre, so musste man Harudes erwarten; denn so lautet der name bei Caesar und auch bei Orosius $(6,7)$ Arudes.

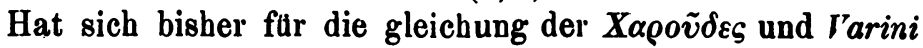
kein unmittelbarer beweis ergeben, so wird sie doch in hohem grade wahrscheinlich, wenn gezeigt werden kann, dass die

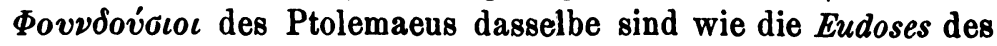
Tacitus. Und das ist in der tat möglich. Unter den hilfsschaaren 
des königs Ariovist werden uns nämlich von Caesar BG. 1, 37 Sedusii und Harudes genannt. Da aber das anlautende $S$ in Sedusii leicht erst aus dem vorausgehenden Nemetes angeschleift ist, und die handschriften des Orosius $(6,7)$, der die stelle nach Caesar widergibt, Eduses, Edures, Eudures bieten, haben schon Zeuss s. 152 und Mullenhoff, Zs. fda. 10, 563 den namen berichtigt. Was dessen flexion betrifft, meint letzterer wohl mit recht, dass sie bei Orosius dem vorangehenden Vangiones, Nemetes gleichgemacht sei. Statt Sedusii hat es also *Eudusii zu heissen, und das ist wesentlich derselbe name wie Eudoses bei Tacitus. Die *Eudusii und Harudes Caesars entsprechen

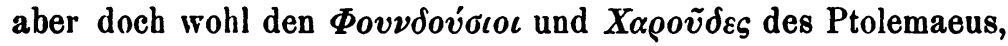

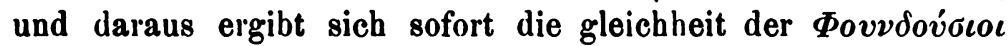

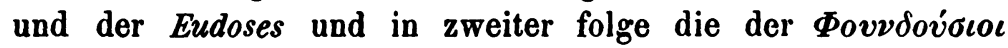

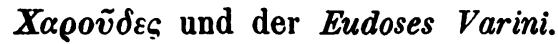

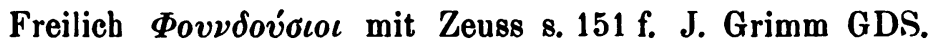

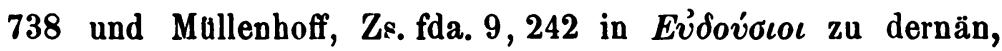
halte ich furr viel zu gewagt. Und warum sollten bier nicht wider zwei verschiedene nur an einander anklingende namen desselben volkes vorliegen?

Eudoses bei Tacitus hat Möller, Beitr. 7, $505 \mathrm{f}$. für Eudoses genommen und als einen eigentumlichen nom. plur. eines $a$ stammes erklärt. Davon wird aber wegen des *Eudusii bei Caesar nicht mehr die rede sein durfen. Vielmehr ist $o$ hier - wie in Gotones Gothones bei 'Tacitus sogar in der stammsilbe - fur germ. $u$ geschrieben. Der name ist also wohl von haus aus ein adjectivischer $s$-stamm, wobei die form mit zutretender $j a$-ableitung vom femininum ihren ausgang nehmen wird.

Gewis hat Mullenhoff, Zs. fda. 10,562 nicht mit unrecht Eudusii mit aisl. jód n. 'proles' zusammengestellt. Wenn dagegen Möller, Beitr. 7, 506 dessen stammsilbe mit der in Jâde (mit fries. iâ aus eu) gleichstellt, so möchte ich doch auch dies nicht zurïckweisen, vielmehr scheinen mir beide ansichten ganz gut vereinbar zu sein. Der name Juthungi im verein mit Eutharicus erweist zur gentige, dass wir von vorgerm. eut, nicht eudh, wie Kremer, Beitr. 8, 437 wollte, auszugehen haben. Das ist aber offenbar dieselbe wurzel, aus der lat. $\bar{u} t e r$ 'schlauch' entsprungen ist, und auf eine andere ablaut- 
stufe derselben wird man ŭterus 'bauch, mutterleib', besonders 'trächtiger mutterleib' zurluck fuhren dürfen. Was die bedeutungsentwickelung anbelangt, ist an das seitenstuck von ags. codd 'schlauch, sack, hulse', aisl. koddi 'polster' und got. qipus, aisl. kviðr, ags. cwið, ahd. quili 'bauch, mutterschoss', ahd. quoden 'femina, interior pars coxae', got. lausqiprs 'nüchtern', aisl. koðri 'tierhode', mnd. koder 'wampe' zu erinnern. Auch der ubergang von dem begriff 'schwangerer mutterleib' $z u$ dem von 'kind' ist leicht begreiflich. Jâde bedeutet also 'sack, schlauch' geradeso wie Codanus, das schon Múllenhoff s. 284 mit ahd. quoden und seiner sippe verglichen hat und nur den lauten nach nicht ganz correct als germ. *quëðn ansetzt; und nm so passender ist ein solcher name fur einen an der seite der breiten Wesermundung gelegenen aber selbst nicht mit einer flussmundung verbundenen meerbusen: vgl. unser sackgasse. Es fragt sich nun, an welche stufe der bedeutungsentwicklung des zu grunde liegenden stammes die ableitung Eudoses Eudusii anknüpft. Es könnte in ihr sehr wohl derselbe begriff enthalten sein wie im namen Juthungi, den wir wegen

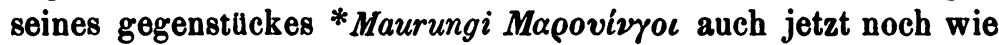
oben 8. 85 als 'die jugendfrischen' verstehen werden. Allein germ. *Eudusez *Eudusjōz zeigt nicht nur eine sehr altertulmliche ableitung, sondern erweist sich auch durch sein $d$ gegenuber dem $b$ in Juthungi und Eutharicus, womit auch aisl. joঠ unmittelbar zusammengehören dürfte, als eine schon in der zeit vor durchfuhrung des germanischen accentes erfolgte wortbildung, und unter solchen umständen ist es wohl gestattet, von einer älteren bedeulung des stammwortes auszugeben. Die *Eudusez *Eudusjōz erscheinen uns dann als 'uterīni', als 'die leiblichen, echten sprösslinge', als ' $\gamma \nu \eta \dot{\sigma} \sigma \iota \iota$ ' oder 'germani'. Dabei beachte man, dass sich in allen diesen worten der begriff des ursprunges aus demselben und dem echten stamme, deinselben uterus, mutterleibe, yévos oder

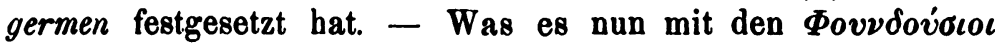
fur eine bewantnis hat, bedarf keiner langen auseinandersetzung: sie sind germanische *Fundusjōz d. i. 'findelkinder'; vgl. ahd. fundaling, mhd. fundelinc oder funtkint, engl. foundling. Denen, die als besonders stammecht gelten wollten, hielt man entgegen, dass sie leute unbekannter herkunft, von den eigenen 
eltern nach der geburt ausgesetzt und von fremden gefunden und aufgezogen worden seien.

Und nun lässt es sich gar nicht mehr verkennen, welche bedeutung gerade bei Ptolemaeus den spott- und ubernamen zukommt, ja dass dessen berichterstatter sie regelmässig bevorzugen, wo mehrere namen einer völkerschaft zur verfügung standen. Das wird vielleicht nicht so sehr in einer tendenz als in der socialen stellung derselben ihren grund hahen. Es ist gewis nicht gleichgiltig, ob ein germanischer füst, ein hoher adeliger, ein heldensänger die quelle ethnographischer nachrichten ist, oder ob sie dem munde eines fahrenden possenreissers, eines händlers, eines soldaten entstammen. Wenn sich heute ein fremder durch einen niederōsterreichischen bäuerlichen viehhändler über die nachbarländer unterrichten lassen wollte, wüde er vielleicht auch statt von Oberösterreichern und Steirern von Mostschädeln und Kropfjodeln zu hören bekommen. -

Fur verwant mit Eudoses hat man ursprünglich allgemein auch den namen Juten genommen: so Zeuss s. 146. J. Grimm GDS. 738 und Mullenhoff, Nordalb. studien 1, 119; wogegen später der gedanke an eine solche verwantschaft, da diese den lautgesetzen widersprãche (s. Möller, Beitr. 7, 506), ebenso allgemein und unbedenklich wider aufgegeben wurde. Und gar nicht mit vollem rechte. Denn zwischen germ. eud- und eutkann ganz gut dasselbe verhältnis bestehen wie etwa zwischen isl. blaùðr, ags. bléad, ahd. blôdi einerseits und mhd. blôz, ags. bléat, aisl. blautr andrerseits, wobei die form mit tenuis auf einer $n$-ableitung bei suffixbetonung beruht: aus vorgerm. eutnomusste germ. eutto und weiterhin euto- euta-werden; vgl. Noreen, Urgerm. judl. § 37, 2. § 39, 3.

Dass der name Juten ursprunglich nicht einen dänischen stamm bezeichnet, sondern einen den Angeln verwanten, erhellt daraus, dass Beda 1, 15 (vgl. Zeuss s. 497) neben Angeln und Sachsen auch Juten unter den germanischen eroberern Britanniens nennt und ihnen die besiedelung von Kent, Wight und Hampshire zuschreibt, wo doch uberall später altenglisch gesprochen wurde. Dieser umstand veranlasste Möller, Ae. volksepos 88 ein von den skadinavischen Jótar (deren namen er als germ. Jeuto-z im nom. sing. ansetzt) verschiedenes volk der Juten in Britannien anzunehmen, das er von den Saxones Eucii 
(d. i. Eutii) und Euthiones herleitet. Letztere sind ihm eine chaukische abteilung, deren reste er ohne weiteres an die niederlāndische kuste setzt, von wo die ubrigen nach Britannien hinubergezogen seien. In wahrheit aber gibt es gar keine Saxones Eucii, da beide namen an der betreffenden stelle in dem briefe des Frankenköniges Theodebert an kaiser Justinian selbständig und einander beigeordnet sind (s. Zeuss s. 387), wie schon daraus hervorgeht, dass sonst der Sachsen daselbst, obwohl sie in einem abhängigkeitsverhältnis zu den Franken standen, gar keine erwähnung geschähe. Ags. Ziotas 3éotas aber, dem zu liebe Möller den namen der dänischen Jótar im altgermanischen mit $j$ anlauten lässt, ist, was seinen anlaut betrifft, entweder so wie zeormen fur eormen yrmen zu beurteilen - wie dies Mullenhoff s. 206 und ten Brink, Beowulf 205 getan haben - oder es ist in dieser gestalt ein lehnwort aus dem dänischen. Jotar braucht also beileibe nicht aut altes *Jeutōz, sondern kann ganz wohl auf *Eutōz oder *Eutonez zurückgehen, wozu jene Eucii (= Eutii) und Euthiones, d. i. *Eutjo $z{ }^{*} E u t j o n e z$, und die $Y^{\prime} t a s, Y^{\prime} t e$ oder $Y^{\prime}$ tan des Widsio trefflich stimmen, da der unterschied der ableitung doch kaum von wesentlicher bedeutung ist. Es werden also Zeuss s. 501 und J. Grimm GDS. $738 \mathrm{im}$ rechte bleiben, wenn sie die Juten fur einen ursprünglieh deutschen stamm halten. Nachdem ein teil desselben nach Britannien thergesiedelt war, baben die tubrigen den Dänen sich unterworfen und mit dänischen einwanderern ihr land geteilt. Von diesen haben sie nach und nach die nordische sprache angenommen, wogegen sie ihren stammnamen auf ihre neuen herrn ubertrugen, doch so, dass diese deshalb den namen Dänen nicht aufgaben, der nur fortan als der weitere erscheint. - Um so grōsser ist unter solchen umstånden die versuchung, den namen der Juten als den eines vordänischen stammes mit dem der Eudoses in zusammenhang zu bringen. Wie uns dann deren andenken der name Jutland forterhält, so finden wir das der Angeln im namen des landes Angeln, das der Haruden im namen des Harsyssel bis auf unsere tage bewahrt.

Aber auch im Widsiðlied zeigten sich alle ingvaeonischen stämme der kimbrischen halbinsel, soweit wir sie bisher kennen

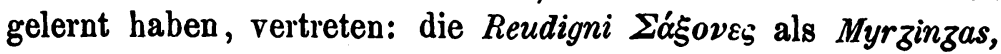




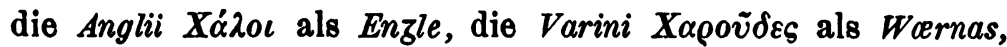

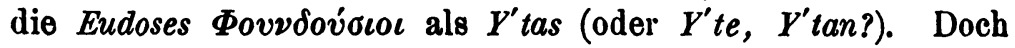
bewahrt das ags. epos mindestens noch einen namen der nach Jutland gehört. Wenn man nur zu dem gen. plur. Wendla im Beowulf 348 und dem dat. plur. Wenlum im Widsið 59 einen nom. Wendle, nicht, wie man bisher getan hat, Wendlas ansetzt, kann dieser name genau dasselbe ausdrücken wie Wendilenses bei Saxo, aisl. Vandilsbyggjar, Vendilfolk, nämlich bewohner von Vendill, dem nördlichsten teile von Jutland bis zum Limfjord: vgl. den norw. volksnamen Firoir 'anwohner des fjords', ags. Myrce 'bewohner der marken'. Auf das einmal bei Saxo vorkommende Wandali wird man sich dabei freilich nicht berufen dürfen, weil das nur antikisierung ist. Naturlich kann Wendla, Wenlum auch zu einem nom. Wendlas = lat. Vandili gehören, also eine form des Vandalennamens sein. Aber was Mullenhoff gegen die seit Grundtvig im norden herschende auffassung der angelsächsischen Wendlen vorbringt, ist kaum stichbaltig. Einen 'amtmann des Vendsyssel' werden wir uns unter dem am hofe des Dänenköniges Hroðgar weilenden Wendla léod Wulfgar nicht vorstellen durfen. Aber kann es nicht der fürst eines von den Dänen unterworfenen und noch nicht in ihnen aufgegangenen ingraeonischen stammes sein? Das liegt doch wohl näher, als dass or von den Wandalen gekommen sei, die zu Hroðgars zeit längst aus dem gesichtskreise der Angeln ausgetreten waren. Warum die auffassung der Wendeln als bewohner des Vendsyssel, wie Mullenhoff, Beovulf 89 meint, voraussetzen wurde, dass Vendill schon zum dänischen reiche gehört habe, ehe die Angelsachsen abzogen, ist mir nicht verständlich. Gegen die Mullenhoffsche annabme spricht auch v. 59 des Widsiðliedes: Mid Wenlum and mid Wornum and mid Wicinzum; denn mit Warnen und Wikingen, d. i. Erulern, zusammen ist doch eher ein drittes von den Nerthusvölkern genannt als ein so entferntes, wie es die Vandalen waren.

Fur die Wendle des Beowulf und Widsio erwarten wir aber bei Tacitus und Ptolemaeus eine entsprechung, und es ist nur in der ordnung, wenn bei diesem tuber den $X \alpha \rho o v \delta \varepsilon \varsigma$ und

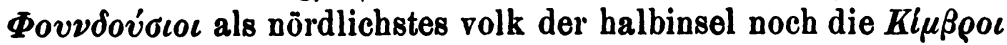
genannt werden. So viel ist auch jetzt schon klar, dass man 
diesen namen bei ihm gar nicht beseitigen könnte, ohne dass eine lücke zurluckbliebe, dass er also nicht erst in folge einer falschen localisierung, wie Mullenhoff dartun wollte, an seine stelle gelangt ist. Im gegenteile muss der eine name $K$ k $\mu \beta \rho o \iota$ den zwei namen Suardones (Suarines) und Nuit(h)ones bei Tacitus die wage halten. Und das mag sogar auffallen. Aber werden nicht auch umgekehrt die Mattiaci des Tacitus durch

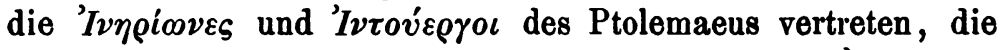

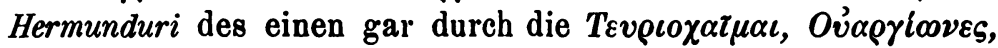

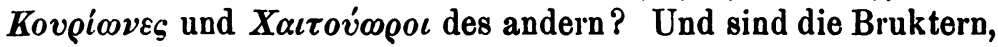
Friesen, Chauken je in zwei unterabteilungen geschieden, warum soll ähnliches nicht auch bei den $K^{\prime} \mu \beta \rho o \iota$ der fall sein? Tacitus und Ptolemaeus lassen sich hier sehr einfach in einklang bringen, wenn man die Suardones (Suarines) und Nuit(h)ones für gau-

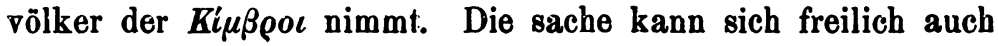
anders verhalten, da es doch nicht völlig ausgeschlossen ist, dass bei Ptolemaeus ein volk libergangen ist. Zuversichtlicher lassen sich daher nur die sätze aufstellen: alle Nuil(h)ones sind Kimbern, alle Wendle sind Kimbern, alle Nuit(h)ones sind Wendle. Ueber das verhältnis der Suardones (Suarines) zu den Kimbern sind zweifel möglich. Und wenn sie selbst zu diesen gehören, ist damit noch nicht gesagt, dass sie auch ein teil der Wendle sind, denn diese können sehr wohl mit den Nuit(h)ones ganz und gar zusammenfallen.

Wenn Möller, Ae. volksepos 6 vorschlägt, statt Nuilhones Vithones zu lesen, und den stamm dieses namens an die Widau (älter $W \hat{\imath} t h \hat{a}$ ) in Schleswig ruckt, liesse sich das nur unter der voraussetzung rechtfertigen, dass die aufzählung der Nerthusvölker bei Tacitus eine ganz ordnungslose ist. Zudem lässt sich der name eines flusses $W \hat{t}$ th $\hat{a}$ allerdings verstehen, jede stutze aber fehlt für die annahme, dass nach einer *Wīpahwo deren anwohner einfach $* W \bar{\imath}$ ponez geheissen haben können. Will man aber umgekehrt den fluss nach dem volke benannt sein lassen, so musste der volksname selbständig erklärt werden können. Was aber sollte ${ }^{*} W \bar{\imath}$ ponez bedeutet haben? Auch scheint es mir gar nicht die beste art zu sein, sich des verderbten Nuithones oder Nuitones zu entledigen, wenn man das anlautende $N$ einfach streicht.

Mit verderbnissen werden wir ja hier sicher rechnen mulssen. 
Und wer kuhn genug ist, mag aus Suardones und Nuitones kurzweg Suartones und Huitones herstellen. Als *Swartonez und *Hwìtonez, 'die schwarzen' und 'die weissen', so etwa nach der verschiedenen farbe ihrer schilde oder ihrer tracht benannt, wären die beiden stämme als gauvölker der Kimbern einleuchtend gerechtfertigt. Und wenn ein par mindere handschriften in der tat huitones bieten, so zeigt das, wie leicht $N$ und $H$ verwechselt werden konnten. Aber Tacitus, der germ. *Hrōflarīks durch Cruptorix widergibt, hätte wohl auch für germ. *Hwĩtonez nicht Huitones sondern Quitones geschrieben. Und schon gar nicht Suardones für *Swartonez; denn auf $\Delta \varepsilon v$ -

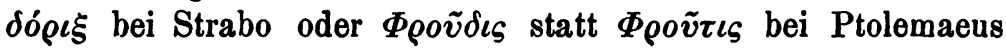
(s. Gluck s. 35) oder Badaus auf einer inschrift (CSL 6, 3240) oder das schwanken der hss. zwischen Aduatuci und Atuatuci darf man sich nicht berufen, weil in sachen der rechtschreibung jeder schriftsteller und jede urkunde aus sich selbst heraus beurteilt werden muss. Wenn dagegen J. Grimm, GDS. 472 die Suardones unter der voraussetzung als 'die schwarzen' gelten lässt, dass dabei, wie ubrigens auch in Eudoses gegentiber Jotar, $d$ noch der dem got. $t$ vorausliegende unverschobene laut sei, so wird ihm darin niemand mehr beipflichten.

Eine andere erklärung, die or s. 471 vorträgt, knlipft nach dem vorgange von Zeuss, s. 154.476 an got. *swaird, ahd. swert an, nach dem die Suardonen so benannt wären wie die Saxonen nach dem sahs. Die Sweordweras des Widsið 62 kämen dieser erklärung naturlich sehr zu statten. Allein für die widergabe von germ. er durch ar findet sich sonst kein beleg, da Arminius lateinisch und Arpus ein germanischer *Arpaz nicht * Erpaz ist: s. Zs. fda. 35, 365. Die zusammenstellung mit ahd. eidswart bei Mullenhoff, Zs. fda. 11, 287 ist schon mit rücksicht auf die grundbedeutung von 'schwören' und auf den mangel des zu erwartenden präfixes $g a$ - wenig ansprechend. Endlich hat gleichfalls aus grunden der bedeutung auch unser schwarte aus dem spiele zu bleiben; denn was wäre mit einem namen des sinnes 'leute mit behaarter haut' anzufangen?

Uebrigens ist es sehr fraglich, ob Suardones die richtige form des namens ist, da die hss. B C c einstimmig Suarines bieten, dem nur in $\mathrm{b}$ vom corrector $\beta$ dones abergeschrieben ist, von den geringeren hss., die zum teil Suardones haben, 
abgesehen. Freilich trägt Suarines den stempel der verderbnis deutlich an sich, da latinisierungen germanischer $n$-stämme nach dem vorbilde von homo hominis nicht ublich sind und es Suarini heissen mlisste, wenn eine $\bar{n} n a$-ableitung wie in Charini oder eine ena-ableitung wie in Varini vorlăge. Die verschiedenheit der lesarten bereitet hier deshalb besondere schwierigkeiten, weil es auf den ersten blick gar nicht begreiflich ist, wie die eine aus der anderen oder beide selbständig aus einer dritten entsprungen sein können. Vielleicht aber verhält sich die sache so, dass Suardiones das ursprüngliche war. Daraus konnte durch tuberspringen des $i$ beim abschreiben einerseits die lesart Suardones entstehen. Eine in einer hs. angebrachte correctur in gestalt eines zwischen $d$ und $o$ ubergeschriebenen $i$ konnte andrerseits leicht als eine correctur für die lautgruppe do aufgefasst, Suardones also bei neuerlicher abschrift durch Suarines widergegeben werden. *Suardiones aber liesse sich als germanisch * Su-ardjonez fassen, das heisst als die schwache form eines bahurrîhiadjectivs, bestehend aus der partikel su 'wohl' und einer $j a$-ableitung, sei es von einem unserem art, mhd. art oder von einem ahd. art f. 'ackerung', alts. ard m. 'wohnort', ags. eard m. 'wohnort, heimat, lage', aisl. ord f. 'ernte, ertrag' entsprechenden urgerm. worte. Die * Suardjonez sind darnach entweder 'die wohlgearteten', wobei als an ein gegenstuck an die keltischen Anartes zu erinnern wäre; oder sie sind 'die gutes ackerland besitzenden'. In letzterem falle wurde sich *Su-ardjonez, was form und inhalt anbelangt, keltischem Suessiones an die seite stellen, das bei Zeuss-Ebel GC.10 als 'bene statuti, locati' erklärt wird: eine deutung, zu der als schlagende bestätigung der hinweis auf Caesar BG. 4 nachzutragen ist, wo die Remen von den Suessionen, ibren nachbarn, berichten: latissimos feracissimosque agros possidere.

Einfacher liegt die sache bei den Nuithones. Mit nurtones, das allein in der hs. $b$ sich findet, und dessen $r$ noch dazu durch den corrector $\beta$ in $i$ gebessert ist, ist nicht viel zu machen, denn den begriff 'die nördlichen' hätten die Germanen sicher nicht durch *nurbonez ausgedrückt; eher noch durfte man dahinter eine ablautform zu dem s. 73 angezogenen aisl. njarð vermuten. Man wird aber von dem weit besser belegten Nuithones oder Nuitones auszugehen haben. Die frage ob $t$ oder 
th das richtige ist, wird nicht so sehr durch den stand der uberlieferung als vielmehr erst durch die etymologie entschieden werden können. Wenn wir im ubrigen an dem namen, wie er auf uns gekommen ist, nichts ändern wollen, mussen wir, da es einen germanischen diphthong $u i$ nicht gibt, Nu-ithones abteilen. Das wäre dann eine bildung nach art von got. *Gibida, *Gipida, Darida, gepid. Fastida, ahd. leilido 'flibrer', was die ursprungliche betonung anbelangt allerdings nur mit letzterem ubereinstimmend. Zum wortstamme halte man ahd. nian, aisl. núa, gnúa neben got. bnauan 'zerreiben'. Aber mit *Nūiponez 'zerreibern' ist doch nichts anzufangen. Und ein verderbnis liegt hier allzu nahe, freilich keines von denen, die man bisher in anschlag gebracht hat. Allein NVITONES kann auf die leichteste weise der welt aus NVTIONES verschrieben worden sein. Germanische *Nutjonez würden sich aus got. nuts (in unnutjans), unserem nütze, mhd. nütze, ahd. nuzzi, ags. nytt. erklären lassen, und um so besser, als das ablautende aisl. nýtr nicht nur 'utilis', sondern auch 'benignus, beneficus, strenuus' bedeutet und widerholt als auszeichnendes beiwort fur personen gebraucht wird. $\mathrm{Ob}$ diese erklärung das richtige trifft oder nicht, ist ubrigens fur den gang unserer untersuchung nicht von belang.

Wohl aber ist ein wort uber den namen Kimbern hier am platze, denn wenn dieser in der tat keltisch wäre, wofür Mullenhoff s. 117 und andere ihn halten, so könnte naturlich ein stamm dieses namens an der nordspitze Jutlands niemals bestanden haben. Wie sich indes aus einem worte cim oder richtiger cimb, das zuerst silber bedeutet haben soll, nach dem glossarium von Cormac aber von dem silber, das den Fomori (seerăubern, Wikingen) als zins (cis) gegeben, auf jeden zins ubertragen wäre, ferner aus cimbid 'vinctus', cimbith 'captivus., cimbidi 'custodias' ein altkeltisches *kimbros 'räuber' mit einiger wahrscheinlichkeit erschliessen oder rechtfertigen lassen soll, ist mir unerfindlich. Wenn tubrigens der name Kimbern nach Festus p. 43 und Plutarch, Marius 11 im gallischen oder germanischen răuber bedeutet haben soll, ist darauf doch nichts oder nicht viel zu geben, weil der name eines volkes, das vielleicht in der heimat schon mit seeraub sich beschäftigte und spăter durch jahre hindurch Gallien brandschatzte, leicht 
erst nachträglich diese appellative hedeutung angenommen hat. Aus ags. cimbinz 'comissura' und seiner sippe, die eine mit

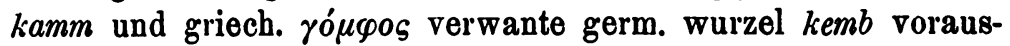
setzt, wird germ. *Kimbrōz freilich nicht erklärt werden dürfen, vielmehr mussen wir uns hiefur um eine wurzel kimb umsehen, die naturlich nur durch nasalierung eines in die $\vec{\imath}$-reihe gehörigen kib entstanden sein kann. Wohl aber entspricht eine zusammenstellung des volksnamens mit isl. kimbi 'spötter', kimbing 'spott' allen anforderungen, da dies zu aisl. kifa 'zanken', kif 'zank', unserem keifen, mhd. kîben, mnld. kîven, ndl. kijven 'rixari', mhd. kîp kîbes 'zänkisches wesen, trotz, widersetzlichkeit' gehört. Und auch die bedeutung 'die zänkischen, die streitsulchtigen', die sich daraus für den volksnamen ergibt, ist so annehmbar, dass ich so wenig wie Tomaschek GGA. 1888 301 einsehen kann, warum Mutlenhoff diese etymologie, die er s. 118 selbst in erwägung zieht, zu gunsten jener so problematischen erklärung aus dem keltischen zurückstellt. Dass der name Cimbri aus dem deutschen, nicht aus dem keltischen zu deuten ist, lehrt auch der name des Srebenherzogs Cimberius, der bei Caesar BG. 1, 37 zusammen mit seinem bruder Nasua namhaft gemacht wird. Nach Mutlenhoff s. $117 \mathrm{f}$. soll dieses Cimberius allerdings nur beweisen, dass der volksname der Kimbern in Deutschlaud nicht unbekannt geblieben war. Allein Cimberius ist doch unmöglich erst eine ableitung aus dem volksnamen, der bei den Germanen nur *Kimbrōz gelautet haben kann, selbst wenn er, was wir damit nicht zugeben wollen, in ihrem munde ein lehnwort gewesen wäre. Vielmehr haben wir es hier mit einer ganz selbständigen bildung aus der wurzel kimb zu tun; und man wird wohl nicht fehl gehen, wenn man deren ableitung fur die urgerm. entsprechung zu ahd. -âri nimmt, zumal auch das aisl. -eri, -ari durch sein erhaltenes $e, a$ auf vorausliegendes $\bar{x}$, also auch auf eine von gotisch -areis abweichende gestalt des suffixes zurlickweist. Sichtbarlich handelt es sich hier um einen beinamen, und *Kimbüerjaz 'der zänker' kann um so eher einer geheissen haben, wenn sein bruder 'der fröhliche' oder 'der ausgelassene' hiess. Nasua, wie es uberliefert ist, weiss ich allerdings nicht zu deuten, denn sicher ist dabei nicht mit Wackernagel, Kleinere schriften 3,350 an nase zu denken, und das ebendort verglichene Nas- 
ualdus auf einer gürtelschnalle, gefunden bei Lavigny im Waadtland, ist Nas-ualdus abzuteilen und in seinem ersten teile mit nisan, nasjan, nara u. s. w. zusammenzustellen: vgl. den ubischen mannesnamen Gasti-nasus oder Gasti-nasius (Brambach, CIRh. 275: Louba Gastinasi filia Ubia). Mit ricksicht auf den Nasua bei Caesar wollte J. Grimm GDS. 493 aus dem Máovos o

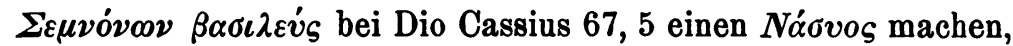
ohne doch daftur eine etymologie beizubringen. Dass aber hier wenigstens $M$ das richtige ist, entscheidet der ahd. mannesname Maso (bei Förstemann DN. 1, 917). Ich wage deshalb umgekehrt fur Nasua bei Caesar Masua in vorschlag zu bringen. Seine erklärung findet germ. *maswaz durch kymr. masw 'hilaris, lascivus, levis'; vgl. auch die keltischen namen Masuco, Masucia, Masuonia, Masunnius, Masuinnus (s. Glick s. 5). Ein lehnwort aus dem keltischen braucht deshalb das germanische wort nicht $\mathrm{zu}$ sein; noch weniger ist naturlich bei dem beinamen eines Semnonenköniges an eine echt keltische benennung zu denken.

Aus der tibereinstimmung der namen $K i \mu \beta \rho o \iota$ und $X \alpha \rho \circ \tilde{v}$ $\delta \varepsilon \varsigma$ bei Ptolemaeus mit Cimbri und Charydes auf dem Monumentum Ancyranum lässt sich widerum das hohe alter der jenem zur verfugung stehenden nachrichten erschliessen. Die quellen des Tacitus sind hier wider die jungeren. Und fand dieser in dem ihm zur verfugung stehenden und von ihm wider. gegebenen verzeichnisse der Nerthusvölker von Kimbern nichts mehr, sondern nur andere namen; war ihm aber aus anderweitigen nachrichten der fortbestand des volkes bekannt und wollte er auf ihre erwăhnung wegen ihrer geschichtlichen bedeutung und eines dabei anzubringenden excurses nicht verzichten, so ist es ganz begreiflich, wenn or aufs geratewohl sie irgendwo unterzubringen suchte und dabei nicht die richtige stelle traf. Ein beweisgrund gegen die glaubwurdigkeit der ubbrigen und ãlteren berichte ist aber daraus bei weitem nicht herzuleiten.

Und was sollte uns sonst wobl bestimmen und berechtigen, alle die zeugnisse vom fortbestehen des kimbrischen volkes zu verwerfen, ja eines davon vom gewichte eines staatsdocumentes geradezu der fälschung zu beschuldigen? In wahrheit zerfliesst alles, was gegen dieselben vorgebracht wurde, in nichts. Denn 
als keltisch lässt sich, wie wir gesehen haben, der name Kimbern nicht rechtfertigen, und der schluss, dass sie, die neben den Teutonen auftreten, nicht auch vom meere kommen können, weil der name Teutonen schon eine keltische gesammtbezeichnung für die nordseevölker sei, hat eine voraussetzung, die sich selbst durch nichts beweisen, wohl aber leicht widerlegen lässt Letzteres ist auch bereits geschehen: vgl. oben s. $5 \mathrm{ff}$.

Wenn die vorstellung von den sitzen der Kimbern in Jutland sammt den aus ihr entspringenden namen $K \iota \mu \beta \rho \iota x \dot{\eta}$ $\chi \varepsilon \varrho \sigma o ́ v \eta \sigma o s$ und promontorium Cimbrorum auf einem irrtum beruhte, so mtisste sich doch ein grund der falschen localisierung angeben oder vermuten lassen. Das ist aber nicht der fall. Denn die keltische flutsage, wenn sie auf die Kimbern ubertragen wäre, wie Mullenhoff s. 166 annimmt, hätte nur zur vorstellung von der herkunft vom ocean im allgemeinen führen, nicht aber die von der herkunft aus einem bestimmten, engbegrenzten bereiche begründen können. Ist aber eine ubertragung der flutsage von den Kelten auf die Kimbern uberhaupt wahrscheinlich? Wenn uns von meeresfluten erzählt wird, die ein nordisches volk zur auswanderung nötigen, so haben wir dabei in anbetracht der bekannten furchtbarkeit der nordseesturmfluten zunächst gar nicht an sage zu denken, und am wenigsten dann, wenn die nachricht von zeitgenossen stammt, oder doch aus einer zeit, in der noch tausende von augenzeugen aller erzählten vorgänge am leben waren. Ueberschwemmungen, zumal solche durch sturmfluten, mochten in der vorzeit mehr als einmal völker zur auswanderung nötigen; dass flutsagen verschiedentlich auftreten, braucht darum nicht oder doch nicht immer aus einer ubertragung sich herzuschreiben. Selbst der langobardischen und der neurischen flutsage, die einstimmig bedrängnis durch schlangen, die hier nur das bekannte mythische bild des wassers sind, als grund der auswanderung angeben, möchte ich nicht kurzweg einen kern von wahrheit absprechen. - Dass im vorletzten jahrzehnte des zweiten vorchristlichen jahrhunderts ein volk aus dem nördlichen Jutland auswanderte, ist um so weniger unwahrscheinlich, als im jahre 58 v. Chr. widerum jutländische Eudusier und Haruden im heere Ariovists in Gallien stehen. 
Dass die Kimbern auch wirklich Deutsche waren, wird man nach dem, was sich uns ther ihre heimat und herkunft bereits ergeben hat, nicht erst zu beweisen brauchen. Es wäre sehr verfehlt, wegen des Keltentumes der Teutonen auch die Kimbern zu Kelten stempeln zu wollen. Im gegenteile müssen sie dann um so eher Deutsche gewesen sein; woher wäre sonst die vorstellung gekommen, dass jene wanderschaaren ganz oder teilweise aus Germanen bestanden haben? An ihrem germanischen volkstum hat auch Mullenhoff nicht gezweifelt und seine bemerkungen uber die erhaltenen kimbrischen königsnamen s. $119 \mathrm{ff}$. sind in allem wesentlichen zu billigen.

Dazu kommt nun noch das zeugnis eines ortsnamens, den man bisher in seiner bedeutung nicht gehörig gewurdigt hat,

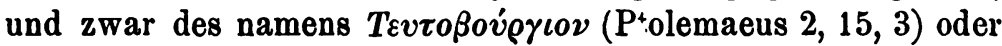
Teutiburgium (Itin. Anton. 243,4) in Pannonien. Ein keltischer name, wofür Mtullenhoff s. 115 ihn hielt, kann das unmöglich sein, weil die keltische entsprechung zu idg. $r$ nicht $u r$, sondern $r i$ und altir. borg 'stadt' erst aus dem altnordischen entlehnt ist. Wir haben es also sicher mit einem germ. * peudaburgja(n) zu tun, einem namen, dessen wortsinn uns schon an die ungeheuren lagerplätze der Kimbern gemahnt, die sich nach Tacitus, Germ. 37 bis in seine zeit erhalten hatten. Denn * Feudaburgja(n) kann nicht nur 'die volksburg', sondern auch 'die grosse burg' bedeuten (vgl. oben s. 74), und es ist wohl auch kein blosser zufall, dass die andere Teutoburg, die, von welcher der Teutoburgiensis saltus den namen fuhrt, zu einer Grotenburg, d. i. 'grossen burg' geworden ist. Um so

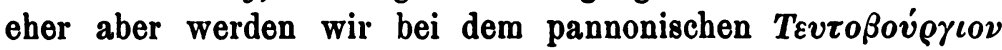
Teutiburgium an ein Kimbernlager denken, als dieser ort hart an der nordgrenze der Skordisken gelegen ist, ebendort also, wo in der richtung des kimbrischen wanderzuges eine wendung eintrat. Man braucht sich den verkehr zwischen den nordleuten und jenem Keltenstamme gar nicht mit Mullenhoff s. 291 als einen feindlichen vorzustellen. Was die Germanen zur umkehr bewogen hat, ist eher als ein für die Kelten gunstiger kampf (von dem uns ja wohl etwas berichtet wüde), die bei diesen eingeholte kunde uber macht und machtbereich der Römer. Sehen wir doch, dass die Germanen selbst nach ibrem siege bei Noreia es vermeiden, in römisches gebiet einzubrechen. 
Auf jeden fall aber setzt der entschluss zur umkehr ein längeres verweilen voraus; und wenn nun eben in der gegend, in der diese rast gehalten worden sein muss, ein germanischer ortsname uns aufstösst, der 'das grosse lager' oder etwas ähnliches bedeutet, so liegt es nahe genug, gerade an der stelle, die er bezeichnet, den wendepunkt der kimbrischen wanderung zu suchen. War der aufenthalt an diesem orte ein längerer, so begreift es sich, dass der name, den die Germanen ihm gaben, auch der einheimischen bevölkerung bekannt wurde. Doch hindert uns auch nichts in der annahme, dass zurlackgebliebene reste der nordischen wanderschaaren ihn der umgebung und den eigenen entnationalisierten nachkommen uberliefert haben.

Erst nachdem die nordjutländische heimat der Kimbern als erwiesen gelten kann, mag zuletzt noch der name Morimarusa hier bertuhrt werden. Plinius berichtet uns HN. 4 \$ 95: septentrionalis Oceanus. Amalcium eum Hecataeus appellat a Parapaniso amne, qua Scythiam adluil; quod nomen eius gentis lingua significat congelatum. Philemon Morimarusam a Cimbris vocari, hoc est mortuum mare, inde usque ad promunturium Rubeas, ultra deinde Cronium. Wenn hier, wie Mullenhoff DA. 1, 413 annimmt, a Cimbris mit inde zu verbinden und bloss örtlich zu verstehen wäre, so hätte sich Plinius schlecht genug ausgedrückt, da jedermann in dem ausdruck a Cimbris vocari zunächst ein gegenstlick $\mathrm{zu}$ dem unmittelbar vorhergehenden eius gentis lingua suchen wird. Freilich sagt Solin 19, 2 bloss: Philemon a Cimbris ad promunturium Rubeas Morimarusam dicit vocari, hoc est mortuum mare; ultra Rubeas quicquid est Cronium nominat. Und somit wird es in der tat fraglich, ob Philemon von einem kimbrischen worte gesprochen hat. Ein solches kann aber trotzdem vorliegen, da es sich um eine örtlichkeit handelt, deren grenze einerseits durch ein zweifellos germanisches, andrerseits durch ein gänzlich unbekanntes local bestimmt wird. Damit soll nicht behauptet werden, dass die Kimbern bereits ausserhalb des gesichtskreises der britannischen und nordgallischen Kelten gelegen haben, dass es also einen keltischen namen fur ein meer, das bis zu den Kimbern hinanreichte, nicht gegeben haben könne. Man bedenke aber, dass Philemon die Kimbern als einen anfangs-, nicht als einen endpunkt namhaft macht. Die möglichkeit, dass wir es bei Morimarusa mit einer ger- 
manischen bezeichnung zu tun haben, hält somit der, dass der name keltisch ist, mindestens die wage, es sei denn, dass die etymologie für die letztere entscheidet. Wirklich fällt Mori

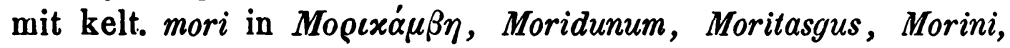
Aremorici, ir. muir, kymr. môr 'meer' vollständig zusammen. Das beweist aber noch gar nichts, da die namen Mainz, Maas, Wasgenwald gegentiber kelt. Moguntiacum, Mosa, Vosegus - vgl. auch got. alên : lat. olīvum, uber das oben s. 34 gehandelt ist zeigen, dass der übergang von idg. $o$ zu germ. $a$ ein verhältnismässig später ist, und wir voraussetzen dürfen, dass im zweiten jahrhundert v. Chr. statt ahd. mari, got. marei noch mori, mori gesprochen wurde. Jedenfalls kann uber die bedeutung von Mori- ein zweifel nicht bestehen. In -marusa muss dann derjenige begriff liegen, den das lat. mortuum widergibt, ja es muss sogar wie dieses ein adjectivum sein, weil es als bestimmungswort einer zusammensetzung an erster stelle zu stehen hätte. Morimarusa ist also für mori marusa zu nehmen, und daraus ergibt sich sofort auch die bedeutungsvolle tatsache, dass mori hier nicht wie ir. muir neutrum, sondern wie got. marei und alts. meri femininum ist. Sicherlich ist marusa mit ir. marb, kymr. marn 'tot' verwant, ist aber doch auch ein ganz anderes wort als dieses. Dagegen haben wir auf germanischer seite bereits ein adjectiv *marsaz und *marzas kennen gelernt, das uns im namen der Marsi und Marsigni sowie in got marzjan u. s. w. erhalten ist. Wenn es nun neben got. marzjan ein aisl. merja gibt, ganz mit der bedeutung unseres mhd. zermürsen, und wenn uns neben mnl. merren, nnl. marren gleichbedeutendes mnl. meren, nnl. meren begegnet, so zeigt sich dabei, dass das $s$ oder $z$ hier geradeso wie etwa in got. talzjan neben untal-s nicht zur wurzel gehört, sondern das einer adjectivischen $s$-ableitung ist, deren ubertritt zur $a$-declination übrigens schon im volksnamen Marsi vollzogen ist. $\mathrm{Zn}$ dem diesem zu grunde liegenden adj. *marsaz aber verhält sich * marusaz wie ags. éar (lat. acus) zu got. ahs, ist also eine nebenform desselben wortes. Wir haben oben s. 112 f. gesehen, dass Franck EW. 614 fur den germ. stamm mars mit recht die bedeutung 'roerloos, talmend, verward' vorausgesetzt hat. Und damit können wir uns auch in dem vorliegenden besonderen falle vollständig zufrieden geben, Denn wenn auch 
Morimarusa eigentlich nur 'das bewegungslose meer' bedeutete, konnte es sehr wohl auch durch mare morluum uersetzt werden. Neben mare mortuum und $\nu \varepsilon x \rho \grave{\eta} \vartheta \alpha \dot{\lambda} \alpha \sigma \sigma \sigma \alpha$ finden sich ja auch die ausdrücke $\pi \varepsilon \pi \eta \gamma v \tau \alpha \vartheta \dot{\alpha} \lambda \alpha \sigma \sigma \alpha$, prope immotum mare, mare pigrum (s. Mullenhoff DA. 1, $410 \mathrm{ff}$.), die, obzwar nicht ausdrticklich als übersetzungen von Morimarusa bezeugt, doch offenbar alle auf denselben barbarischen begriff und namen zurlickgehen, oder allenfalls auf einen germanischen und einen sinnverwanten keltischen. Wenn das mit germ. *marwaz urverwante ir. marb, kymr. marn 'tot' bedeutet, so ist es ubrigens gar nicht ausgeschlossen, dass auch das derselben wurzel entstammende und mit germ. *marwaz, wie wir s. 112 gesehen haben, synonyme germ. *marsaz von der bedeutung 'unbeweglich' aus diejenige von 'leblos' erreicht hatte. Umgekehrt darf man wohl für das kelt. *maruos in älteren sprachperioden neben der bedeutung 'tot' auch noch die beim germ. *marwaz festgehaltene voraussetzen. Germanischem *morī marusā, jünger *marī *marusō, kann also sehr gut ein gleichbedeutendes keltisches *mori *maruon entsprochen haben.

WIEN, im winter 1891/92.

RUDOLF MUCH.

\section{Berichtigungen und nachtrage.}

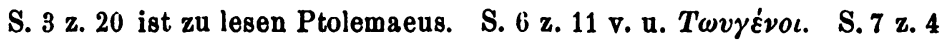

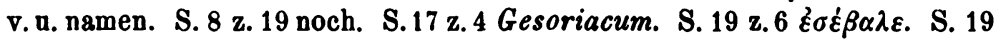
z. 13 v. u. $\S 170$ [statt: §6i]. S. 21 z. 14 magnitudine.... hanc. S. 25 z. 7 der mittelpunkt des. S. 32 z. 7 v. u. lüž (ans *lŭgiñ). S. 33 z. 10 (wie Mitllenhoff. S. 35 z. 10 gradaugaben. S. 38 z. 17 v. u. ist. S. 40 z. 20 stelle [statt: stelle des]. S. 40 z. 14 v. u. Angliorum. S. 43 z. 12 im Beowulf. S. 55 z. 19 v. u. $\dot{v} \varphi$ '. S. 61 anm. z. 16 v. u. von germ. $h$.

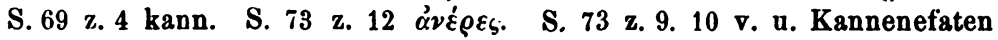

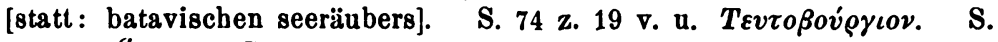
76 z. 1 ó $\pi \omega \varsigma$ s. $\quad$ S. 81 z. 17 lta. $\quad$ S. 83 z. 10 der Sachsen. S. 85 z. 8 v. u. *Mauringōz, ebendort *Naristōz. S. 88 z. 10 v. u. Quorum. S. 89

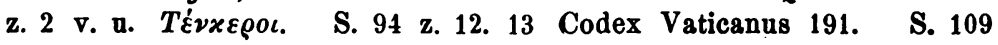
z. 13 Gesenke. S. 113 z. 15 v. u. zwischen. S. 117 z. 5 isl. [statt: aisl.] S. 120 z. 8 Rondinzas. S. 122 z. $20 \varsigma^{\prime \prime}$ [statt: $\varsigma^{\prime \prime}$ ]. S. 124 z. 5 Racu-

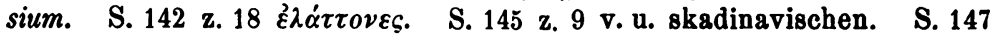
z. 20 Friesen. S. 147 z. 1 v. u. Römerzeit. S. 157 z. 19.20 etymologien des namens. S. 157 z. 22 unternahm. S. 168 z. 5 v. u. 'trans Rhenum' 